JOURNAL OF THE

AMERICAN MATHEMATICAL SOCIETY

Volume 25, Number 4, October 2012, Pages 929-958

S 0894-0347(2012)00737-1

Article electronically published on May 1, 2012

\title{
THREE-POINT BOUNDS FOR ENERGY MINIMIZATION
}

\author{
HENRY COHN AND JEECHUL WOO
}

\section{INTRODUCTION}

Consider the seven lines connecting opposite vertices of a cube and of its dual octahedron. Although the symmetry group does not act transitively on the lines, they are exceedingly well distributed within $\mathbb{R P}^{2}$. In this paper, we prove that they form a universally optimal configuration; in other words, they minimize a wide variety of natural notions of energy. Universal optima are rare, and we show that this configuration is the largest universal optimum in $\mathbb{R P}^{2}$.

To prove universal optimality, we use semidefinite programming bounds, which are a powerful technique for proving bounds in coding theory. We give a new derivation of these bounds and extend them from coding to energy minimization. Proving universal optimality involves challenges that have not arisen in previous applications of semidefinite programming bounds, and we provide a general methodology for meeting these challenges. Furthermore, we conjecture that in certain other cases our bounds remain sharp throughout a phase transition between two different ground states, which would be a remarkable phenomenon. We have not yet been able to prove these conjectures, but the techniques we introduce here represent the first steps in a program to do so.

1.1. Background. What does it mean to distribute $N$ points as uniformly as possible in a compact metric space $X$ with metric $d$ ? There are many possible answers, such as forming a good error-correcting code, i.e., maximizing the distance between the closest points. One particularly important family of answers generalizing coding theory is given by potential energy minimization. Given a decreasing, continuous function $f:\left(0, \max _{x, y \in X} d(x, y)^{2}\right] \rightarrow \mathbb{R}$, called the potential function, define the energy of a finite configuration $\mathcal{C} \subseteq X$ by

$$
E_{f}(\mathcal{C})=\frac{1}{2} \sum_{\substack{x, y \in \mathcal{C} \\ x \neq y}} f\left(d(x, y)^{2}\right) .
$$

Note that the use of squared distance instead of distance is not standard in physics, but it is mathematically convenient (and of course there is no loss of generality). We wish to choose $\mathcal{C}$ so as to minimize $E_{f}(\mathcal{C})$ subject to $|\mathcal{C}|=N$. Because $f$ is decreasing, this amounts to moving the points as far apart as possible, but all the distances matter, not just the minimal distance.

Received by the editors March 2, 2011 and, in revised form, February 24, 2012.

2010 Mathematics Subject Classification. Primary 05B40, 52A40, 52C17; Secondary 90C22, $82 \mathrm{~B} 05$.

The second author was supported in part by an internship at Microsoft Research New England and by a Samsung Scholarship. 
This energy minimization problem arises naturally in physics, as the problem of determining the ground state of a classical particle system with isotropic pair interactions. Even in the case of particles confined to two-dimensional surfaces, these models capture important features of many real-world materials, such as colloidal particles adsorbing to the surface of a droplet (see BG09] for details and other examples).

Small systems frequently display beautiful symmetry, such as twelve particles on a sphere forming the vertices of an icosahedron, but in larger systems the symmetry is often broken by the appearance of defects. One might expect that these defects would occur only in local minima for energy, and that the global minimum would have a perfect crystalline structure, with a large symmetry group. Sometimes that is the case, but in many systems the defects actually contribute to lowering energy. (See for example the discussion of spherical crystals in Section 3 of [BG09].) One of the fundamental problems in this area is understanding when highly symmetrical configurations are optimal and why; see C10 for a survey. Even when the answer is easy to guess, it is usually not easy to prove, and guessing the answer can itself be tricky.

Energy minimization can also be viewed as a generalization of coding theory. Specifically, it includes coding theory as a degenerate special case: if we take $f(r)=$ $1 / r^{s}$, then as $s \rightarrow \infty$, the problem of minimizing $E_{f}(\mathcal{C})$ turns into the problem of maximizing the minimal distance in $\mathcal{C}$. (Ties are broken by how many times this distance occurs, then what the next smallest distance is, then how many times it occurs, etc.) The same problem of understanding symmetry occurs here as well: when should one expect an optimal code to be highly symmetrical? Large or highdimensional codes are frequently much less highly structured than small codes.

Linear programming bounds are a powerful technique for proving lower bounds for energy. In the simplest cases, they deal with the distance distribution of the configuration (in physics terms, the radial pair correlation function), which counts how many times each distance occurs between pairs of points. In other words, the distance distribution of $\mathcal{C}$ is the function $\delta:[0, \infty) \rightarrow \mathbb{R}$ defined by

$$
\delta(r)=|\{x, y \in \mathcal{C}: d(x, y)=r\}| .
$$

Clearly $\delta(0)=|\mathcal{C}|, \delta(r) \geq 0$ for all $r$, and $\sum_{r} \delta(r)=|\mathcal{C}|^{2}$ (note that only finitely many terms are nonzero), but we will see that these are far from the only constraints on $\delta$. The distance distribution plays a key role because energy is a linear function of $\delta$ :

$$
E_{f}(\mathcal{C})=\frac{1}{2} \sum_{r>0} f\left(r^{2}\right) \delta(r) .
$$

In contrast with its energy, the underlying configuration $\mathcal{C}$ is not always determined by $\delta$ (see $\mathrm{P} 44$ ).

Delsarte [D72] realized that in addition to the obvious constraints on $\delta$ mentioned above, there are often many other linear constraints. Using these constraints, one can derive bounds on energy via linear programming, because one is optimizing a linear function of $\delta$ subject to linear constraints. This was first carried out for energy minimization by Yudin Y92. We refer to them as two-point bounds because they depend only on the distances between pairs of points. Linear programming bounds are generally not sharp, or even close to sharp, at least as best we can judge based on the available evidence. However, in certain cases they are unexpectedly powerful. For example, Cohn and Kumar [CK07] used linear programming bounds 
to prove that a number of exceptional structures in spheres or projective spaces are universally optimal. In other words, these configurations minimize energy for all completely monotonic functions of squared Euclidean distance on spheres or squared chordal distance in projective space. (Recall that a completely monotonic function is a smooth, nonnegative function whose derivatives alternate in sign: it is decreasing, convex, etc. For example, inverse power laws are completely monotonic. See [CK07, p. 101] for motivation for the use of squared chordal distance, and [CK07, pp. 107-108] for counterexamples to a natural generalization.) Examples include the vertices of any regular polytope with simplicial facets, the $E_{8}$ root system, or the minimal vectors in the Leech lattice.

It is surprising that linear programming bounds are ever sharp, because when they are sharp, pair distance information alone suffices to identify the true ground state. As one might expect, that is very rarely the case. To prove stronger bounds, it is natural to try to take into account triples as well as pairs (i.e., how many times each triangle of distances occurs between three points), and Schrijver S05 found the right approach. The constraints are no longer linear, but rather semidefinite. Bachoc and Vallentin BV08, developed a representation-theoretic explanation and extended the method from binary codes to spheres, with an approach that applies also to more general spaces, and this method was further developed by Musin [M07. These three-point semidefinite programming bounds are one of the most powerful general tools known for proving bounds in coding theory. Using them, Bachoc and Vallentin BV09b determined the optimal 10-point code in $S^{3}$, which was the first new optimality proof for a spherical code in decades. They also conjectured that the bounds are sharp for the optimal 8-point code in $S^{2}$ (a square antiprism, first proved optimal by Schütte and van der Waerden [SW51, so there was less motivation to verify the sharpness in this case).

1.2. Our results. In this paper, we give a new derivation of the semidefinite programming bounds, which has the advantage of requiring no explicit calculation beyond what is necessary for the linear programming bounds. We then prove semidefinite bounds for potential energy minimization. Using three-point bounds, we prove universal optimality for a seven-point code in $\mathbb{R P}^{2}$. It is given by the seven lines through opposite vertices of a cube and its dual octahedron. Equivalently, the lines connect opposite vertices of a rhombic dodecahedron. In the dual picture of planes through the origin in $\mathbb{R}^{3}$, the planes are parallel to the facets of a cuboctahedron (the dual polyhedron to the rhombic dodecahedron).

Theorem 1. The rhombic dodecahedron code is universally optimal in $\mathbb{R P}^{2}$. It is globally minimal for energy for each completely monotonic potential function of squared chordal distance, and it is the unique global minimum unless the potential function is a linear function. It is also the unique optimal seven-point code in $\mathbb{R P}^{2}$.

It is straightforward to check that two-point bounds cannot prove Theorem 1. It was already known that this configuration is an optimal projective code (i.e., it maximizes the minimal distance), as a consequence of the orthoplex bound of Conway, Hardin, and Sloane CHS96. Uniqueness was conjectured in CHS96 but does not follow from the orthoplex bound; see Appendix A for an explanation.

Note that the uniqueness asserted in Theorem 1 is as strong as possible: for linear potential functions, uniqueness fails because one can rotate the cube and octahedron relative to each other (so they are no longer in dual position) without 
changing the energy. That can be checked directly, but conceptually it holds because both polyhedra already define projective 1-designs. More generally, $k$-designs automatically minimize potential energy for completely monotonic polynomials of degree at most $k$.

One noteworthy aspect of the rhombic dodecahedron code is that it is the first case in which linear or semidefinite programming bounds are sharp for a code that is not distance regular. In other words, there is a distance such that the points in the code do not all have the same number of neighbors at that distance. All universal optima known or conjectured so far have been distance regular, so this example is the least regular one known. Theorem 1 thus extends the types of configurations that can be analyzed rigorously.

We have searched extensively for other cases in which the three-point semidefinite programming bounds are sharp (in spheres, real projective spaces, and complex projective spaces), but the rhombic dodecahedron is the only new case we have found. It would be very surprising if it were the only example in projective space, and we think there must be others. However, our failure to find them suggests that they are either large or high-dimensional. In the process of searching for other examples, we have formulated several conjectures about semidefinite programming bounds that would be remarkable if true (Conjectures 13 through 15): in the few sharp cases that are known, the bounds appear to remain sharp even all the way through a phase transition between two different structures.

Theorem 1 provides the last remaining universal optimum in $\mathbb{R P}^{2}$ :

Theorem 2. The complete list of universally optimal line configurations in $\mathbb{R}^{3}$ (up to isometry) is as follows:

(1) Up to three orthogonal lines.

(2) The four lines through opposite vertices of a cube.

(3) The six lines through opposite vertices of an icosahedron.

(4) The seven lines through opposite vertices of a rhombic dodecahedron.

Case (11) is trivial, while cases (2) and (3) follow from Theorem 8.2 in [CK07. Case (4) is Theorem [1, and the completeness of the list is proved in Appendix B]

1.3. Notation and definitions. A code is simply a finite subset of a metric space. It is optimal if it maximizes the minimal distance between points, given the number of points and the metric space.

An $(n, N, t)$ spherical code is a set of $N$ points in the unit sphere $S^{n-1}$ such that the maximal inner product between distinct points is at most $t$. (In other words, the minimal angle between them is at least $\cos ^{-1} t$.) An antipodal code is a spherical code that is closed under multiplication by -1 . Real projective codes are of course equivalent to antipodal codes, and the rhombic dodecahedron code corresponds to a $(3,14,1 / \sqrt{3})$ antipodal code. (Note that the vertices of the rhombic dodecahedron do not lie on a common sphere, so they must be scaled to form this code.)

The chordal metric is defined on real, complex, or quaternionic projective space as follows (in the octonionic case it is defined using the Frobenius norm on the exceptional Jordan algebra; see [CK07, p. 130]). If we represent points in projective space by unit vectors, then the chordal distance between $x$ and $y$ is $\sqrt{1-|\langle x, y\rangle|^{2}}$, where $\langle x, y\rangle$ is the Hermitian inner product. This metric, first introduced in [CHS96], is equivalent to the Fubini-Study metric but is in many ways more convenient. 
A code in a sphere or projective space is universally optimal if it minimizes the energy $E_{f}$ for all completely monotonic potential functions $f$ (compared to all other codes of the same size), with the metric chosen to be the Euclidean metric on spheres or the chordal metric on projective spaces. Recall that distance is squared in the definition of $E_{f}$. This strengthens the notion of universal optimality, compared to defining it with unsquared distance, and it improves the connections with topics such as spherical or projective designs. (See [CK07, p. 101].)

Let $P_{k}^{n}(t)$ denote the degree $k$ ultraspherical (i.e., Gegenbauer) polynomial for $S^{n-1}$, normalized with $P_{k}^{n}(1)=1$. (This is not the most common notation.) These polynomials are orthogonal with respect to the measure $\left(1-t^{2}\right)^{(n-3) / 2} d t$ on $[-1,1]$. See Chapters 6 and 9 of AAR99 for more details.

In the literature, two-point bounds are typically referred to as linear programming bounds and three-point bounds as semidefinite programming bounds. These names are a reasonable reference to the underlying inequalities, but we propose calling them $k$-point bounds, which focuses attention on the most geometrically relevant feature, namely the number of points being simultaneously considered.

Given a matrix $A$, we write $A \succeq 0$ if $A$ is Hermitian (i.e., it equals its own conjugate transpose) and positive semidefinite. The inner product on Hermitian matrices is defined by $\langle A, B\rangle=\operatorname{tr}(A \bar{B})$ (i.e., the entry-by-entry Hermitian inner product), and angle brackets will also denote the usual inner product on vectors. We will use $J$ to denote a matrix with all entries 1 .

When we deal with points in real projective space $\mathbb{R} \mathbb{P}^{n-1}$, we will represent each point by an arbitrary lift to the double cover $S^{n-1}$. That introduces sign ambiguities, so we must ensure that all formulas are invariant under the choice of the lift. For example, if we apply a function to the inner product between two points, then it should be an even function.

\section{LiNEAR AND SEMIDEFINITE PROGRAMMING BOUNDS}

2.1. Positive-definite kernels. Linear and semidefinite programming bounds are based on the theory of positive-definite kernels. Let $G$ be a topological group acting continuously on a topological space $X$. Call a continuous function $K: X \times X \rightarrow \mathbb{C}$ a positive-definite kerne 1 if for all $N \in \mathbb{N}$ and all $x_{1}, \ldots, x_{N} \in X$, the $N \times N$ matrix $\left(K\left(x_{i}, x_{j}\right)\right)_{1 \leq i, j \leq N}$ is Hermitian and positive semidefinite. (Of course, saying it is Hermitian simply means that $K(x, y)=\overline{K(y, x)}$ for all $x, y \in X$.) We call the kernel $G$-invariant if $K(g x, g y)=K(x, y)$ for all $g \in G$ and $x, y \in X$.

Given any unitary representation $V$ of $G$ with inner product $\langle\cdot, \cdot\rangle$ and any continuous map $\varphi: X \rightarrow V$ that is $G$-equivariant (i.e., $\varphi(g x)=g \varphi(x)$ for $g \in G$ and $x \in X)$, setting $K(x, y)=\langle\varphi(x), \varphi(y)\rangle$ defines a $G$-invariant positive-definite kernel on $X$. In fact, every such kernel arises in this way:

Theorem 3. For every $G$-invariant positive-definite kernel $K$ on $X$, there exists a unitary representation $V$ of $G$ and a continuous, $G$-equivariant map $\varphi: X \rightarrow V$ such that $K(x, y)=\langle\varphi(x), \varphi(y)\rangle$ for all $x, y \in X$.

This theorem is a variant of a characterization of positive-definite kernels due to Bochner B41]. See, for example, $\S 5$ of Chapter IV in [L85] for a closely related result (with the same idea behind it).

\footnotetext{
${ }^{1}$ In an ideal world, we would call $K$ a positive-semidefinite kernel, but unfortunately this terminology is too well established to be easily changed.
} 
Proof. Let $W$ be the complex vector space formally spanned by the points of $X$, and define a form $\langle\cdot, \cdot\rangle$ on $W$ by setting $\langle x, y\rangle=K(x, y)$ for $x, y \in X$ and extending linearly in the first coordinate and conjugate linearly in the second. The action of $G$ on $X$ extends to an action of $G$ on $W$.

Because $K$ is a $G$-invariant positive-definite kernel, the form $\langle\cdot, \cdot\rangle$ on $W$ is $G$ invariant and positive semidefinite. Let $W_{0}$ be the subspace of vectors that have norm 0 . Then the inner product on $W / W_{0}$ is positive definite, and the completion $V$ of $W / W_{0}$ is a Hilbert space. Let $\varphi: X \rightarrow V$ be the obvious map (the composition of the trivial embedding of $X$ in $W$, the quotient map by $W_{0}$, and the embedding in the completion). Then $\varphi$ is continuous and $G$-equivariant, and $K(x, y)=\langle\varphi(x), \varphi(y)\rangle$ for all $x, y \in X$.

To complete the proof, we need only verify that $V$ is a unitary representation of $G$. In other words, for each $v \in V$, the map $g \mapsto g v$ must be a continuous function from $G$ to $V$. By $G$-invariance it suffices to verify continuity at $g=1$. Thus, we wish to show that $g v \rightarrow v$ as $g \rightarrow 1$. Furthermore, it suffices to show this convergence for a dense subset of $V$, and we choose $W / W_{0}$ as that subset. Therefore, we can assume that

$$
v=\sum_{x \in X} c_{x}[x]
$$

where $[x]$ denotes the vector in $V$ corresponding to $x \in X$ and where all but finitely many of the coefficients $c_{x}$ vanish. Then

$$
|g v-v|^{2}=\sum_{x, y \in X} c_{x} \overline{c_{y}}(2 K(x, y)-K(x, g y)-K(g x, y)) .
$$

By the continuity of $K$ and the action of $G$ on $X$, the finitely many terms on the right side with nonvanishing coefficients can be made arbitrarily small by making $g$ close to the identity. Thus, $V$ is indeed a unitary representation of $G$.

If $V$ splits into an orthogonal direct sum $V=V_{1} \oplus V_{2}$ as a representation of $G$, then each corresponding kernel $K$ splits as $K=K_{1}+K_{2}$, where $K_{1}$ and $K_{2}$ correspond to the subrepresentations $V_{1}$ and $V_{2}$.

Suppose that $G$ is compact. Then by the Peter-Weyl theorem, every unitary representation of $G$ is an orthogonal direct sum of finite-dimensional irreducible representations. Thus, the cone of $G$-invariant positive-definite kernels is spanned by those arising from irreducible representations.

When $G$ acts transitively on $X$, we can identify $X$ with $G / H$, where $H$ is the stabilizer of a point $e \in X$. Then a $G$-equivariant map $\varphi: X \rightarrow V$ is completely determined by $\varphi(e)$ via $\varphi(g e)=g \varphi(e)$, and $\varphi(e)$ can be any vector in $V$ that is fixed by $H$.

The simplest case is when $(G, H)$ is a Gelfand pair: then the fixed space $V^{H}$ has dimension at most 1 when $V$ is irreducible, so each irreducible representation is associated with at most one positive-definite kernel (up to scaling). This occurs, for example, when $X$ is a sphere, projective space, or Grassmannian and $G$ is the group of isometries of $X$.

When the fixed space $V^{H}$ has dimension greater than 1 , the situation is more complicated. Then $V$ is associated with several positive-definite kernels, but in fact there is a richer structure behind them. For $x_{1}, x_{2} \in X$, define a sesquilinear form 
$K_{x_{1}, x_{2}}$ on $V^{H}$ as follows. Let $x_{i}=g_{i} e$ with $g_{1}, g_{2} \in G$, and for $v_{1}, v_{2} \in V^{H}$ define

$$
K_{x_{1}, x_{2}}\left(v_{1}, v_{2}\right)=\left\langle g_{1} v_{1}, g_{2} v_{2}\right\rangle \text {. }
$$

The key property of these forms is that for all $N \in \mathbb{N}, x_{1}, \ldots, x_{N} \in X$, and $v_{1}, \ldots, v_{N} \in V^{H}$,

$$
\sum_{1 \leq i, j \leq N} K_{x_{i}, x_{j}}\left(v_{i}, v_{j}\right) \geq 0
$$

To prove this inequality, note that

$$
\sum_{1 \leq i, j \leq N} K_{x_{i}, x_{j}}\left(v_{i}, v_{j}\right)=\sum_{1 \leq i, j \leq N}\left\langle g_{i} v_{i}, g_{j} v_{j}\right\rangle=\left|\sum_{1 \leq i \leq N} g_{i} v_{i}\right|^{2} .
$$

As a consequence (by taking $v_{1}=\cdots=v_{N}$ ), for all $N \in \mathbb{N}$ and $x_{1}, \ldots, x_{N} \in X$,

$$
\sum_{1 \leq i, j \leq N} K_{x_{i}, x_{j}} \succeq 0
$$

where $A \succeq 0$ means that $A$ is positive-semidefinite and Hermitian. The $G$-invariant positive-definite kernels are given by $(x, y) \mapsto K_{x, y}(v, v)$ with $v \in V^{H}$, but the underlying structure of $K$ is of broader importance.

In general, define a matrix-valued positive-definite kernel to be a map that takes $x, y \in X$ to a sesquilinear form $K_{x, y}$ on a fixed complex vector space $W$ (not necessarily finite-dimensional), with the following three properties:

(1) For all $v, w \in W$, the map $(x, y) \mapsto K_{x, y}(v, w)$ is continuous.

(2) For all $x, y \in X$ and $v, w \in W$,

$$
K_{x, y}(v, w)=\overline{K_{y, x}(w, v)} .
$$

(3) For all $N \in \mathbb{N}$, all $x_{1}, \ldots, x_{N} \in X$, and all $w_{1}, \ldots, w_{N} \in W$,

$$
\sum_{1 \leq i, j \leq N} K_{x_{i}, x_{j}}\left(w_{i}, w_{j}\right) \geq 0
$$

The last two properties are equivalent to requiring that

$$
\left(K_{x_{i}, x_{j}}\left(w_{i}, w_{j}\right)\right)_{1 \leq i, j \leq N} \succeq 0 .
$$

We say that $K$ is defined on $X$ and over $W$. The matrix-valued kernel is $G$ invariant if $K_{g x, g y}=K_{x, y}$ for all $g \in G$ and $x, y \in X$. The construction above defines a $G$-invariant matrix-valued positive-definite kernel over $V^{H}$ whenever $G$ acts transitively on $X$. Note that when $\operatorname{dim} W=1$, a matrix-valued positivedefinite kernel over $W$ is the same as an ordinary (scalar-valued) kernel.

When $G$ does not act transitively on $X$, the identification of the vector space $\operatorname{Hom}_{G}(X, V)$ of continuous, $G$-equivariant maps with $V^{H}$ breaks down. Nevertheless, essentially the same construction works. One can define a $G$-invariant matrix-valued positive-definite kernel over $\operatorname{Hom}_{G}(X, V)$ by setting

$$
K_{x_{1}, x_{2}}\left(\varphi_{1}, \varphi_{2}\right)=\left\langle\varphi_{1}\left(x_{1}\right), \varphi_{2}\left(x_{2}\right)\right\rangle
$$

for $x_{1}, x_{2} \in X$ and $\varphi_{1}, \varphi_{2} \in \operatorname{Hom}_{G}(X, V)$. This construction is fully general, aside from changing variables in trivial ways: 
Theorem 4. For every $G$-invariant matrix-valued positive-definite kernel $K$ on $X$ and over $W$, there exists a unitary representation $V$ of $G$ and a linear function $\varphi: W \rightarrow \operatorname{Hom}_{G}(X, V)$ (written $w \mapsto \varphi_{w}$ ) such that for all $x, y \in X$ and $v, w \in W$,

$$
K_{x, y}(v, w)=\left\langle\varphi_{v}(x), \varphi_{w}(y)\right\rangle .
$$

Proof. Let $U=W \otimes_{\mathbb{C}} \mathbb{C} X$, where $\mathbb{C} X$ denotes the complex vector space formally spanned by the points of $X$. We define a form $\langle\cdot, \cdot\rangle$ on $U$ by

$$
\left\langle\sum_{i} v_{i} \otimes x_{i}, \sum_{j} w_{j} \otimes x_{j}\right\rangle=\sum_{i, j} K_{x_{i}, x_{j}}\left(v_{i}, w_{j}\right)
$$

for $v_{i}, w_{i} \in W$ and $x_{i} \in X$. Because $K$ is a matrix-valued positive-definite kernel, this defines a Hermitian, positive-semidefinite form on $U$. Let $U_{0}$ denote the subspace of vectors with norm 0 , and let $V$ be the completion of $U / U_{0}$, so $V$ is a Hilbert space. Define $\varphi_{w}(x)$ to be the image of $w \otimes x$ in $V$.

The Hilbert space $V$ is a unitary representation of $G$, with the trivial action of $G$ on $W$ and the usual action on $X$. (Strong continuity follows as in the proof of Theorem 3]) By construction,

$$
K_{x, y}(v, w)=\left\langle\varphi_{v}(x), \varphi_{w}(y)\right\rangle,
$$

which completes the proof.

When $X=S^{n-1}$ and $G$ is the stabilizer in $O(n)$ of a point in $X$, the matrices constructed in Theorem 3.1 of [BV08] (by a different method from that used here) define matrix-valued positive-definite kernels, and we were led to our construction in an attempt to develop a more abstract approach to Bachoc and Vallentin's discovery.

2.2. Linear and semidefinite programming bounds. Given the machinery of positive-definite kernels, it is easy to write down linear and semidefinite programming bounds for energy minimization. We will write $X^{2} / G$ for the set of orbits of $G$ acting on pairs, and $[x, y]$ will denote the orbit of the pair $(x, y)$.

The linear programming bounds involve linear constraints on the G-invariant pair distribution of a code. Given a finite subset $\mathcal{C} \subseteq X$, define

$$
A_{x, y}=\left|\left\{(u, v) \in \mathcal{C}^{2}:[u, v]=[x, y]\right\}\right| .
$$

These numbers satisfy some obvious linear constraints. They are all nonnegative, and their sum over the set $X^{2} / G$ of orbits is $|\mathcal{C}|^{2}$. Furthermore, $A_{x, x}=|\mathcal{C}|$ if $G$ acts transitively on $X$, and even if that is not the case, the diagonal terms $A_{x, x}$ are the orbit sizes of $G$ acting on $X$.

In addition to these obvious constraints, each $G$-invariant positive-definite kernel $K: X \times X \rightarrow \mathbb{C}$ gives a more subtle constraint. Specifically,

$$
\sum_{[x, y] \in X^{2} / G} A_{x, y} K(x, y)=\sum_{x, y \in \mathcal{C}} K(x, y) \geq 0 .
$$

Of course, the sum over $[x, y] \in X^{2} / G$ means a sum over representatives of the orbits. Positive-definite kernels are remarkable because they have this property despite typically being negative at many points. Pfender [P07] discovered that they are not the only such functions, and one can occasionally improve the linear programming bounds by incorporating his functions. Unfortunately the improvement seems to be small in general, and the method becomes much less systematic 
(because the representation-theoretic context is lost), so we will not use Pfender's approach here.

Given a $G$-invariant potential function $f: X \times X \rightarrow \mathbb{R}$, define the energy of $\mathcal{C}$ by

$$
\frac{1}{2} \sum_{\substack{x, y \in \mathcal{C} \\ x \neq y}} f(x, y)=\frac{1}{2} \sum_{\substack{[x, y] \in X^{2} / G \\ x \neq y}} A_{x, y} f(x, y) .
$$

Potential energy is a linear functional of the pair distribution $A$, so by solving a linear programming problem (possibly infinite dimensional) one can optimize it subject to the linear constraints described above. To prove a lower bound for energy, valid for arbitrary sets of $|\mathcal{C}|$ points in $X$, one need only find a feasible point in the dual linear program.

When $X=G / H$ and $(G, H)$ is a Gelfand pair, linear programming bounds express all systematically available information about the pair distribution. In more general cases, linear programming bounds can be generalized to two-point semidefinite programming bounds. They work the same way, except they use the matrix-valued positive-definite kernels $K_{x, y}$ developed in Subsection 2.1 For each such kernel,

$$
\sum_{[x, y] \in X^{2} / G} A_{x, y} K_{x, y} \succeq 0,
$$

which is a semidefinite constraint on $A$. Thus, optimizing energy subject to these constraints becomes a semidefinite programming problem.

2.3. Three-point bounds and beyond. The most important application of semidefinite programming bounds is to prove three-point bounds (as in BV08]). Fix a point $e \in X$ and let $H=\operatorname{Stab}_{G}(e)$ be its stabilizer. The semidefinite programming bounds give constraints on $H$-invariant pair distributions on $X$, and symmetrizing $e$ with the other two points turns them into constraints on $G$-invariant triple distributions. One can then attempt to optimize energy (or other quantities) subject to these constraints.

Of course, we are not limited to using just three points, and one can prove $k$-point bounds in the same way. (Musin [M07] was the first to formulate these bounds for spherical codes.) However, as $k$ increases the bounds become increasingly difficult to compute with. The difficulty is that $k$-point distributions are functions on $X^{k} / G$, so $k$-point bounds involve optimization over functions on this space. When $X^{k} / G$ is one-dimensional, the optimization problem is usually doable, but it rapidly becomes intractable as the dimension increases.

For a concrete example, suppose $X=S^{n-1}$ and $G=O(n)$. Then elements of $X^{k} / G$ are determined by their pairwise distances, so $\operatorname{dim} X^{k} / G=\left(\begin{array}{c}k \\ 2\end{array}\right)$. For $k=3$, functions of three variables are barely tractable, but for $k=4$, functions of six variables are too complicated: the space of polynomials of degree at most $m$ in six variables has dimension $\left(\begin{array}{c}m+6 \\ 6\end{array}\right)$, which grows too rapidly to allow for extensive computations.

The situation can be much worse for other spaces. For example, a pair of points in $\mathbb{C P}^{n-1}$ is determined up to isometries by the distance between them, but for triples of points there is a fourth parameter, namely a complex phase change. (Two unit vectors in $\mathbb{C}^{n}$ can easily be phase shifted so that their inner product is real, but for three vectors that is generally impossible.) The Grassmannian of 
$k$-dimensional subspaces in $\mathbb{R}^{n}$ is even worse, since $\min (k, n-k)$ parameters are required to determine a pair up to isometries (see, for example, [B06]).

2.4. Explicit computations. Of course, to apply any of these bounds in practice, one must carry out the representation-theoretic computations explicitly. Fortunately, in the cases of interest in this paper, no more calculations are needed to set up $k$-point bounds than two-point bounds.

Recall that for the sphere $S^{n-1}$, it is a theorem of Schoenberg $\mathbf{S} 42$ that the cone of $O(n)$-invariant positive-definite kernels is spanned by the functions $(x, y) \mapsto$ $P_{k}^{n}(\langle x, y\rangle)$. Here, $P_{k}^{n}$ denotes the $k$-th degree Gegenbauer polynomial for $S^{n-1}$. See Subsection 2.2 of [CK07] for a brief account of these calculations. In terms of Theorem 3 these positive-definite kernels correspond to the irreducible representations of $O(n)$ that contain a nonzero vector fixed by $O(n-1)$ (and the other irreducible representations do not have corresponding kernels).

Once this characterization of positive-definite kernels is known, one can extend it to $k$-point bounds without needing any additional representation theory or information about special functions. For example, three-point bounds can be handled as follows. This gives a new proof of Corollary 3.5 in BV08.

Theorem 5. Let $H$ be the stabilizer in $O(n)$ of a point e in $S^{n-1}$. For each $k \geq 0$, there is an $H$-invariant matrix-valued positive-definite kernel on $S^{n-1}$ that takes $x_{1}, x_{2} \in S^{n-1}$ to the infinite matrix whose $\left(i_{1}, i_{2}\right)$-entry (indexed starting with 0 ) is

$$
u_{1}^{i_{1}} u_{2}^{i_{2}}\left(\left(1-u_{1}^{2}\right)\left(1-u_{2}^{2}\right)\right)^{k / 2} P_{k}^{n-1}\left(\frac{t-u_{1} u_{2}}{\sqrt{\left(1-u_{1}^{2}\right)\left(1-u_{2}^{2}\right)}}\right),
$$

where $u_{j}=\left\langle e, x_{j}\right\rangle$ and $t=\left\langle x_{1}, x_{2}\right\rangle$.

Of course, for numerical computations one uses finite submatrices of these infinite matrices.

Proof. Let $X=S^{n-1}$, with $e$ and $H$ as in Theorem 5. We must compute matrixvalued positive-definite kernels on the vector space $\operatorname{Hom}_{H}(X, V)$, where $V$ is an irreducible unitary representation of $H$. Of course, $\operatorname{Hom}_{H}(X, V)$ is infinite dimensional, but we will choose a basis for a dense subset.

Let

$$
Y=\left\{y \in S^{n-1}:\langle e, y\rangle=0\right\}
$$

be the equator relative to $e$. To specify a map $\varphi \in \operatorname{Hom}_{H}(X, V)$, we just need to specify its restriction to the equator and to all the parallel slices of the sphere. Fortunately, that is relatively manageable. As in $\operatorname{Subsection}_{2.1} \operatorname{Hom}_{H}(Y, V) \cong$ $V^{H}$ because $H$ acts transitively on $Y$, and the Gelfand pair property implies that $V^{H}$ is at most one-dimensional; the same is true for all the parallel slices. Thus, the map $\varphi$ is determined by specifying a scaling factor on each slice, which is a function of one variable.

Suppose $\operatorname{dim} V^{H}=1$, and choose an element $\tilde{\varphi} \in \operatorname{Hom}_{H}(Y, V)$ that is not identically zero. We will use $\tilde{\varphi}$ to define $\varphi$ on the equator $Y$. To complete the determination of $\varphi$, we must specify the scaling factor on each parallel slice. We will use scaling factors that are polynomials in the inner product with $e$ (up to multiplication by some fixed function $f$ to be specified later). Specifically, for $-1 \leq u \leq 1$ and $y \in Y$, define

$$
\varphi_{i}\left(u e+\sqrt{1-u^{2}} y\right)=u^{i} f(u) \tilde{\varphi}(y) .
$$


The function $\varphi_{i}$ is $H$-equivariant because $\tilde{\varphi}$ is, and as $i$ varies these functions are dense in $\operatorname{Hom}_{H}(X, V)$.

To compute the matrix-valued positive-definite kernel, we need to compute $\left\langle\varphi_{i_{1}}\left(x_{1}\right), \varphi_{i_{2}}\left(x_{2}\right)\right\rangle$ for $x_{1}, x_{2} \in X$. If we write $x_{j}=u_{j} e+\sqrt{1-u_{j}^{2}} y_{j}$, then

$$
\left\langle\varphi_{i_{1}}\left(x_{1}\right), \varphi_{i_{2}}\left(x_{2}\right)\right\rangle=u_{1}^{i_{1}} u_{2}^{i_{2}} f\left(u_{1}\right) f\left(u_{2}\right)\left\langle\tilde{\varphi}\left(y_{1}\right), \tilde{\varphi}\left(y_{2}\right)\right\rangle .
$$

The inner product $\left\langle\tilde{\varphi}\left(y_{1}\right), \tilde{\varphi}\left(y_{2}\right)\right\rangle$ can be computed using Schoenberg's theorem, because $Y$ is a sphere and $H$ is the full isometry group of $Y$. Specifically, there is some $k$ (depending on $V$ ) such that

$$
\left\langle\tilde{\varphi}\left(y_{1}\right), \tilde{\varphi}\left(y_{2}\right)\right\rangle=P_{k}^{n-1}\left(\left\langle y_{1}, y_{2}\right\rangle\right)
$$

if $\tilde{\varphi}$ is rescaled appropriately, which we can assume.

If we let $t=\left\langle x_{1}, x_{2}\right\rangle$, then

$$
\left\langle y_{1}, y_{2}\right\rangle=\frac{t-u_{1} u_{2}}{\sqrt{\left(1-u_{1}^{2}\right)\left(1-u_{2}^{2}\right)}} .
$$

It follows that the $\left(i_{1}, i_{2}\right)$-entry $\left\langle\varphi_{i_{1}}\left(x_{1}\right), \varphi_{i_{2}}\left(x_{2}\right)\right\rangle$ of the matrix-valued positivedefinite kernel is

$$
u_{1}^{i_{1}} u_{2}^{i_{2}} f\left(u_{1}\right) f\left(u_{2}\right) P_{k}^{n-1}\left(\frac{t-u_{1} u_{2}}{\sqrt{\left(1-u_{1}^{2}\right)\left(1-u_{2}^{2}\right)}}\right) .
$$

It is convenient to choose $f(u)=\left(1-u^{2}\right)^{k / 2}$. Then

$$
\left\langle\varphi_{i_{1}}\left(x_{1}\right), \varphi_{i_{2}}\left(x_{2}\right)\right\rangle=u_{1}^{i_{1}} u_{2}^{i_{2}}\left(\left(1-u_{1}^{2}\right)\left(1-u_{2}^{2}\right)\right)^{k / 2} P_{k}^{n-1}\left(\frac{t-u_{1} u_{2}}{\sqrt{\left(1-u_{1}^{2}\right)\left(1-u_{2}^{2}\right)}}\right) .
$$

This choice has the advantage that the right side is a polynomial in $u_{1}, u_{2}$, and $t$ (because $P_{k}^{n-1}$ is an even or odd function, according as $k$ is even or odd).

The same approach works straightforwardly for $k$-point bounds: choosing $H$ to be the stabilizer of several points recovers the results of Musin [M07. It also works for projective spaces. As in the case of spheres, the calculations for an $n$-dimensional projective space with respect to the stabilizer of a point reduce immediately to those for an $(n-1)$-dimensional space with respect to the full group.

The calculations in real projective space are almost the same as those in the sphere. If we lift points arbitrarily to the sphere (as discussed in Subsection 1.3), then we just need to avoid any sign ambiguity. Specifically, in terms of the three inner products $u_{1}, u_{2}$, and $t$ from Theorem 5. we want only terms that are invariant under changing the signs of two of the three variables. That means we take only the entries for which $i_{1} \equiv i_{2} \equiv k(\bmod 2)$. This submatrix defines a matrix-valued positive-definite kernel on real projective space.

\section{THREE-POINT BOUNDS FOR ENERGY MINIMIZATION}

In this section, we write down three-point bounds for energy minimization in spheres or real projective spaces. We begin with spheres, after which we can easily adapt the answer to real projective spaces. All of the required theory is in Section 2 , but there are a number of details that must be worked out carefully. 
It will be convenient to write potential functions in terms of the inner product. Given a potential function $f:[-1,1) \rightarrow \mathbb{R}$, define

$$
\widetilde{E}_{f}(\mathcal{C})=\frac{1}{2} \sum_{\substack{x, y \in \mathcal{C} \\ x \neq y}} f(\langle x, y\rangle) .
$$

Because $|x-y|^{2}=2-2\langle x, y\rangle$, if we set $f(t)=g(2-2 t)$, then $\widetilde{E}_{f}(\mathcal{C})=E_{g}(\mathcal{C})$. The function $f$ is absolutely monotonic (i.e., it is infinitely differentiable and all of its derivatives are nonnegative) if and only if $g$ is completely monotonic. Thus, $\mathcal{C}$ is universally optimal if and only if it minimizes $\widetilde{E}_{f}$ for all absolutely monotonic $f$.

Given a configuration $\mathcal{C} \subseteq S^{n-1}$ with $|\mathcal{C}|=N>2$, define the corresponding triple distribution by

$$
A_{u, v, t}=\left|\left\{(x, y, z) \in \mathcal{C}^{3}:\langle x, y\rangle=u,\langle y, z\rangle=v,\langle z, x\rangle=t\right\}\right| .
$$

This function counts ordered triples of points modulo the action of $O(n)$. Of course, $A_{u, v, t} \geq 0$, and $A_{u, v, t}=0$ unless $-1 \leq u, v, t \leq 1$ and

$$
1+2 u v t-u^{2}-v^{2}-t^{2} \geq 0 .
$$

In other words, the $3 \times 3$ Gram matrix

$$
\left(\begin{array}{lll}
1 & u & v \\
u & 1 & t \\
v & t & 1
\end{array}\right)
$$

must be positive semidefinite. Furthermore, $A_{u, v, t}$ is symmetric in $u, v$, and $t$. It satisfies the identities $A_{1,1,1}=N$,

$$
\begin{aligned}
& \sum_{u} A_{u, u, 1}=N^{2}, \\
& \sum_{u, v, t} A_{u, v, t}=N^{3},
\end{aligned}
$$

and

$$
\sum_{v, t} A_{u, v, t}=N A_{u, u, 1}
$$

Define

$$
D=\left\{(u, v, t):-1 \leq u, v, t<1 \text { and } 1+2 u v t-u^{2}-v^{2}-t^{2} \geq 0\right\} .
$$

Because the potential function is not necessarily defined at inner product 1, it will be important to work with sums over $D$. For example,

$$
\begin{aligned}
\sum_{(u, v, t) \in D} A_{u, v, t} & =\sum_{u, v, t} A_{u, v, t}-3 \sum_{u} A_{u, u, 1}+2 A_{1,1,1} \\
& =N^{3}-3 N^{2}+2 N \\
& =N(N-1)(N-2) .
\end{aligned}
$$

Equivalently, summing over $D$ counts ordered triples of distinct points. 
We can express $\widetilde{E}_{f}(\mathcal{C})$ as a sum over $D$ by writing

$$
\begin{aligned}
\widetilde{E}_{f}(\mathcal{C}) & =\frac{1}{2} \sum_{u<1} A_{u, u, 1} f(u) \\
& =\frac{1}{2 N} \sum_{u<1, v, t} A_{u, v, t} f(u) \\
& =\frac{1}{2 N}\left(2 \sum_{u<1} A_{u, u, 1} f(u)+\sum_{(u, v, t) \in D} A_{u, v, t} f(u)\right) \\
& =\frac{1}{2 N}\left(4 \widetilde{E}_{f}(\mathcal{C})+\sum_{(u, v, t) \in D} A_{u, v, t} f(u)\right) .
\end{aligned}
$$

It follows that

$$
\widetilde{E}_{f}(\mathcal{C})=\frac{1}{6(N-2)} \sum_{(u, v, t) \in D} A_{u, v, t}(f(u)+f(v)+f(t)) .
$$

Let $S_{k}^{n}(u, v, t)$ be the infinite matrix whose $(i, j)$-entry (indexed starting with 0 ) is the symmetrization of

$$
u^{i} v^{j}\left(\left(1-u^{2}\right)\left(1-v^{2}\right)\right)^{k / 2} P_{k}^{n-1}\left(\frac{t-u v}{\sqrt{\left(1-u^{2}\right)\left(1-v^{2}\right)}}\right)
$$

in $u, v$, and $t$ (i.e., the average over all permutations of the variables). The sum of this matrix over the code is positive semidefinite by Theorem 5 , in terms of $A_{u, v, t}$,

$$
\sum_{u, v, t} A_{u, v, t} S_{k}^{n}(u, v, t) \succeq 0 .
$$

If we break the sum up according to which variables equal 1, we find that

$$
\sum_{(u, v, t) \in D} A_{u, v, t} S_{k}^{n}(u, v, t)+3 \sum_{u<1} A_{u, u, 1} S_{k}^{n}(u, u, 1)+N S_{k}^{n}(1,1,1) \succeq 0 .
$$

(It might appear that $S_{k}^{n}(u, v, t)$ is undefined when one of the variables equals 1 , but in fact all its entries are polynomials in $u, v$, and $t$.) The same trick as we used for $\widetilde{E}_{f}$ shows that $\sum_{u<1} A_{u, u, 1} S_{k}^{n}(u, u, 1)$ equals

$$
\frac{1}{3(N-2)} \sum_{(u, v, t) \in D} A_{u, v, t}\left(S_{k}^{n}(u, u, 1)+S_{k}^{n}(v, v, 1)+S_{k}^{n}(t, t, 1)\right) .
$$

(In fact, this is simply (3.1) with $f(u)$ replaced with $2 S_{k}^{n}(u, u, 1)$ ) Thus, if we define

$$
T_{k}^{n}(u, v, t)=(N-2) S_{k}^{n}(u, v, t)+S_{k}^{n}(u, u, 1)+S_{k}^{n}(v, v, 1)+S_{k}^{n}(t, t, 1),
$$

then

$$
N(N-2) S_{k}^{n}(1,1,1)+\sum_{(u, v, t) \in D} A_{u, v, t} T_{k}^{n}(u, v, t) \succeq 0 .
$$


Furthermore, note that $S_{k}^{n}(1,1,1)$ is the zero matrix unless $k=0$, in which case all of its entries are 1 . If we let $J$ denote the all 1 matrix, then our inequality becomes

$$
N(N-2) \delta_{k, 0} J+\sum_{(u, v, t) \in D} A_{u, v, t} T_{k}^{n}(u, v, t) \succeq 0,
$$

where $\delta$ denotes the Kronecker delta function.

Theorem 6. The minimal value of $\widetilde{E}_{f}$ for $N>2$ points in $S^{n-1}$ is greater than or equal to the optimum of the following semidefinite program: minimize

$$
\frac{1}{6(N-2)} \sum_{(u, v, t) \in D} A_{u, v, t}(f(u)+f(v)+f(t))
$$

over all choices of $A_{u, v, t} \geq 0$ for $(u, v, t) \in D$, subject to

$$
\sum_{(u, v, t) \in D} A_{u, v, t}=N(N-1)(N-2)
$$

and

$$
N(N-2) \delta_{k, 0} J+\sum_{(u, v, t) \in D} A_{u, v, t} T_{k}^{n}(u, v, t) \succeq 0
$$

for $k \geq 0$.

To compute a lower bound for energy, we will use the dual semidefinite program. Suppose we define a function $H$ on $D$ by

$$
H(u, v, t)=c+\sum_{k \geq 0}\left\langle F_{k}, T_{k}^{n}(u, v, t)\right\rangle,
$$

where $c$ is a constant, $F_{k}$ denotes an infinite symmetric matrix, and the inner product on symmetric matrices is the trace of the product. We assume only finitely many entries of $F_{k}$ are nonzero, so the inner product is well defined.

Theorem 7. With the notation established above, if $F_{k} \succeq 0$ for all $k$ and

$$
H(u, v, t) \leq \frac{f(u)+f(v)+f(t)}{3}
$$

for all $(u, v, t) \in D$, then the minimal value of $\widetilde{E}_{f}$ for $N$ points in $S^{n-1}$ is at least

$$
\frac{N}{2}\left((N-1) c-\left\langle F_{0}, J\right\rangle\right)
$$

Proof. For any $N$-point code $\mathcal{C}$ with triple distribution $A$,

$$
\sum_{(u, v, t) \in D} A_{u, v, t} H(u, v, t) \leq 2(N-2) \widetilde{E}_{f}(\mathcal{C})
$$

by (3.1). On the other hand, the inner product of two positive semidefinite matrices is nonnegative, from which it follows that

$$
N(N-2)\left\langle F_{k}, J\right\rangle \delta_{k, 0}+\sum_{(u, v, t) \in D} A_{u, v, t}\left\langle F_{k}, T_{k}^{n}(u, v, t)\right\rangle \geq 0 .
$$

Summing over $k$ yields

$$
N(N-2)\left\langle F_{0}, J\right\rangle+\sum_{(u, v, t) \in D} A_{u, v, t}(H(u, v, t)-c) \geq 0,
$$

and combining these inequalities with $\sum_{(u, v, t) \in D} A_{u, v, t}=N(N-1)(N-2)$ completes the proof. 
As discussed at the end of Section 2, the real projective case is almost identical. If $f$ is an even function, then we can lift points arbitrarily to $S^{n-1}$ without introducing any ambiguity in $\widetilde{E}_{f}$. It will prove convenient to use a third variant $\widehat{E}$ of the notation for energy (in addition to $E$ and $\widetilde{E}$ ); that may seem unnecessary, but $E$ has the clearest connection to physics, $\widetilde{E}$ is the most convenient for spheres, and $\widehat{E}$ is the most convenient for real projective space. Given a potential function $f:[0,1) \rightarrow \mathbb{R}$, define

$$
\widehat{E}_{f}(\mathcal{C})=\frac{1}{2} \sum_{\substack{x, y \in \mathcal{C} \\ x \neq y}} f\left(\langle x, y\rangle^{2}\right) .
$$

Here $x$ and $y$ represent lifts to $S^{n-1}$ of points in $\mathbb{R} \mathbb{P}^{n-1}$. Note that each point in $\mathbb{R} \mathbb{P}^{n-1}$ has two lifts, but we only include one of them in the sum (chosen arbitrarily).

Let $\widehat{S}_{k}^{n}$ be the submatrix of $S_{k}^{n}$ indexed by numbers with the same parity as $k$, and define

$$
\widehat{T}_{k}^{n}(u, v, t)=(N-2) \widehat{S}_{k}^{n}(u, v, t)+\widehat{S}_{k}^{n}(u, u, 1)+\widehat{S}_{k}^{n}(v, v, 1)+\widehat{S}_{k}^{n}(t, t, 1) .
$$

Then the three-point energy bounds for $\mathbb{R P}^{n-1}$ are exactly the same as those for $S^{n-1}$, except with $\widehat{S}$ and $\widehat{T}$ replacing $S$ and $T$ :

Theorem 8. If

$$
H(u, v, t)=c+\sum_{k \geq 0}\left\langle F_{k}, \widehat{T}_{k}^{n}(u, v, t)\right\rangle
$$

with $F_{k} \succeq 0$ for all $k$ and

$$
H(u, v, t) \leq \frac{f\left(u^{2}\right)+f\left(v^{2}\right)+f\left(t^{2}\right)}{3}
$$

for all $(u, v, t) \in D$, then the minimal value of $\widehat{E}_{f}$ for $N$ points in $\mathbb{R} \mathbb{P}^{n-1}$ is at least

$$
\frac{N}{2}\left((N-1) c-\left\langle F_{0}, J\right\rangle\right) .
$$

The choice of $c$ and $F_{0}, F_{1}, \ldots$ in Theorems $[7$ and 8 can be optimized using semidefinite programming. First, assume $F_{k}=0$ for $k$ beyond some bound, and that for all $k$ the $(i, j)$-entry of $F_{k}$ vanishes if $i$ and $j$ are sufficiently large, so that only finitely many variables need to be considered. Assume also that $f$ is a polynomial (although that assumption can be relaxed, at the cost of additional complications). To express the constraint that

$$
H(u, v, t) \leq \frac{f\left(u^{2}\right)+f\left(v^{2}\right)+f\left(t^{2}\right)}{3}
$$

for $(u, v, t) \in D$, we will use a sum of squares representation due to Putinar. Unfortunately, there is a nontrivial error in the paper [P93] (see [M08, p. 98]), so we must be careful here. The space $D$ is defined by the constraints $1-u^{2} \geq 0$, $1-v^{2} \geq 0,1-t^{2} \geq 0$, and $1+2 u v t-u^{2}-v^{2}-t^{2} \geq 0$, and this representation of $D$ is stably compact (i.e., it defines a compact set even if the coefficients are perturbed); furthermore, only the last constraint has odd degree. It follows from Corollary 7.2.5 in M08] that if a polynomial is strictly positive on $D$, then it can be expressed in the form

$$
\begin{aligned}
G_{0}(u, v, t) & +G_{1}(u, v, t)\left(1-u^{2}\right)+G_{2}(u, v, t)\left(1-v^{2}\right)+G_{3}(u, v, t)\left(1-t^{2}\right) \\
& +G_{4}(u, v, t)\left(1+2 u v t-u^{2}-v^{2}-t^{2}\right)
\end{aligned}
$$


where $G_{0}(u, v, t), \ldots, G_{4}(u, v, t)$ are sums of squares of polynomials in $u, v, t$. A polynomial is a sum of squares if and only if it is of the form $\langle z(u, v, t), M z(u, v, t)\rangle$, where $M$ is a symmetric, positive-semidefinite matrix and $z(u, v, t)$ is a vector whose entries are monomials in $u, v, t$; thus, being a sum of squares is a semidefinite condition. If we apply this approach to

$$
\frac{f\left(u^{2}\right)+f\left(v^{2}\right)+f\left(t^{2}\right)}{3}-H(u, v, t)
$$

then we get semidefinite programs that are guaranteed to come arbitrarily close to the true optimum in Theorem 7 Of course, we hope they will actually achieve the true optimum, and in practice that occurs.

\section{Proof of Universal optimality}

In this section we prove Theorem 1, There are two fundamental difficulties. One is that, although we can solve a semidefinite program numerically to obtain a bound for any given potential function, the solutions are very cumbersome and it is not easy to produce a rigorous, exact solution. Bachoc and Vallentin dealt with this difficulty in BV09b, but their problem is substantially simpler and they employed ad hoc techniques. We will develop a more systematic approach.

The second difficulty is that we must prove optimality for infinitely many different potential functions, namely all completely monotonic functions. Of course we only need to deal with the extreme rays in this cone, but there are infinitely many of them as well. To address this difficulty, we will construct a finite set of functions (not all completely monotonic) such that optimality for all of them implies universal optimality. In other words, we will replace the cone of completely monotonic functions with a larger cone that has only finitely many extreme rays, so we will prove a slightly stronger result than universal optimality.

Recall that

$$
\widehat{E}_{f}(\mathcal{C})=\frac{1}{2} \sum_{\substack{x, y \in \mathcal{C} \\ x \neq y}} f\left(\langle x, y\rangle^{2}\right),
$$

where $x$ and $y$ represent lifts to $S^{n-1}$ of points in $\mathbb{R P}^{n-1}$ (with only one lift of each point being used). One advantage of squaring the inner product is that it becomes invariant under sign changes, but it also relates well to the chordal distance. The squared chordal distance between $x$ and $y$ is $1-\langle x, y\rangle^{2}$, so $\widehat{E}_{f}(\mathcal{C})=E_{g}(\mathcal{C})$ with $g(t)=f(1-t)$. Thus, $\mathcal{C}$ is universally optimal in $\mathbb{R} \mathbb{P}^{n-1}$ if and only if it minimizes $\widehat{E}_{f}$ for each absolutely monotonic function $f$. The cone of absolutely monotonic functions on $[0,1)$ is spanned by the monomials $f(t)=t^{i}$ (see Theorem $9 \mathrm{~b}$ in W41, p. 154]), so it suffices to prove optimality for these monomials.

To reduce proving universal optimality to a finite problem, we will apply Hermite interpolation. Recall that given a nonempty, finite multiset $T$ of points in $\mathbb{R}$ (with the multiplicity of $t \in T$ denoted $\operatorname{mult}_{T}(t)$ ), the Hermite interpolation $\mathcal{H}_{T}(f)$ of a function $f$ is the unique polynomial of degree less than

$$
\sum_{t \in T} \operatorname{mult}_{T}(t)
$$


that agrees with $f$ to order mult $T(t)$ at each $t \in T$. (I.e., $f^{(i)}(t)=\left(\mathcal{H}_{T}(f)\right)^{(i)}(t)$ for all $i<\operatorname{mult}_{T}(t)$.) See Subsection 2.1 of CK07 for background on Hermite interpolation. The following observation of Yudin will be crucial:

Lemma 9 (Yudin [Y92]). Let $T$ be a finite, nonempty multisubset of an interval $I$ such that each point in $T$ has even multiplicity, except for the endpoints of I (which are allowed to have even or odd multiplicity). For each absolutely monotonic function $f: I \rightarrow \mathbb{R}$,

for all $t \in I$.

$$
\mathcal{H}_{T}(f)(t) \leq f(t)
$$

Lemma 9 follows from the remainder formula for Hermite interpolation (see, for example, Lemmas 2.1 and 5.1 in [CK07] for a proof).

Lemma 10. Let $T=\left\{t_{1}, \ldots, t_{M}\right\}$ be a nonempty multisubset of an interval $I$ (written with $t_{i}$ repeated according to its multiplicity). If $f: I \rightarrow \mathbb{R}$ is absolutely monotonic, then $\mathcal{H}_{T}(f)$ is a nonnegative linear combination of the partial products

$$
t \mapsto \prod_{i=1}^{m}\left(t-t_{i}\right)
$$

for $0 \leq m<M$. (Of course, the $m=0$ case is the constant function 1 .)

What Lemma 10 says depends on the ordering of $t_{1}, \ldots, t_{M}$, but it is true for every ordering.

Proof. We prove Lemma 10 by induction on $M$. For $M=1, \mathcal{H}_{T}(f)$ is the constant function $f\left(t_{1}\right)$ and the lemma is trivial. Otherwise, let $T^{\prime}=\left\{t_{2}, \ldots, t_{M}\right\}$. Then

$$
\left(\mathcal{H}_{T}(f)\right)(t)=f\left(t_{1}\right)+\left(t-t_{1}\right)\left(\mathcal{H}_{T^{\prime}}(g)\right)(t),
$$

where $g(t)=\left(f(t)-f\left(t_{1}\right)\right) /\left(t-t_{1}\right)$ (and $\left.g\left(t_{1}\right)=f^{\prime}\left(t_{1}\right)\right)$. The function $g$ is absolutely monotonic on $I$, by Proposition 2.2 in CK07]; alternatively, that can be seen directly via

$$
\frac{d^{k}}{d t^{k}}\left(\frac{f(t)-f\left(t_{1}\right)}{t-t_{1}}\right)=\frac{\int_{t_{1}}^{t} f^{(k+1)}(u)\left(u-t_{1}\right)^{k} d u}{\left(t-t_{1}\right)^{k+1}},
$$

which is the fundamental theorem of calculus for $k=0$ and can be proved by induction (or by using a Taylor series expansion for $\left(f(t)-f\left(t_{1}\right)\right) /\left(t-t_{1}\right)$ about $\left.t_{1}\right)$. Now applying the lemma inductively to $\mathcal{H}_{T^{\prime}}(g)$ and using (4.1) completes the proof.

Combining Lemmas 9 and 10 reduces proving universal optimality to a finite number of cases, as follows.

Corollary 11. Let $\mathcal{C}$ be a finite subset of $\mathbb{R P}^{n-1}$ (represented via arbitrary lifts to $S^{n-1}$ ), and let $T=\left\{t_{1}, \ldots, t_{M}\right\}$ be any finite multisubset of $[0,1)$ (written with multiplicities) such that all elements other than 0 have even multiplicity and

$$
\left\{\langle x, y\rangle^{2}: x, y \in \mathcal{C}, x \neq y\right\} \subseteq T .
$$

If $\mathcal{C}$ minimizes $\widehat{E}_{f_{m}}$ for the potential functions

$$
f_{m}(t)=\prod_{i=1}^{m}\left(t-t_{i}\right)
$$

with $0 \leq m<M$, then $\mathcal{C}$ is universally optimal in $\mathbb{R} \mathbb{P}^{n-1}$. 
Proof. Let $f:[0,1) \rightarrow \mathbb{R}$ be absolutely monotonic. By Lemma 9 , $f \geq \mathcal{H}_{T}(f)$ on $[0,1)$, and by Lemma 10, there are nonnegative coefficients $\lambda_{0}, \ldots, \lambda_{M-1}$ such that

$$
\mathcal{H}_{T}(f)=\lambda_{0} f_{0}+\cdots+\lambda_{M-1} f_{M-1} .
$$

Thus, for any configuration $\mathcal{D}$ with $|\mathcal{D}|=|\mathcal{C}|$,

$$
\begin{aligned}
\widehat{E}_{f}(\mathcal{D}) & \geq \widehat{E}_{\mathcal{H}_{T}(f)}(\mathcal{D}) \\
& =\lambda_{0} \widehat{E}_{f_{0}}(\mathcal{D})+\cdots+\lambda_{M-1} \widehat{E}_{f_{M-1}}(\mathcal{D}) \\
& \geq \lambda_{0} \widehat{E}_{f_{0}}(\mathcal{C})+\cdots+\lambda_{M-1} \widehat{E}_{f_{M-1}}(\mathcal{C}) \\
& =\widehat{E}_{\mathcal{H}_{T}(f)}(\mathcal{C}) \\
& =\widehat{E}_{f}(\mathcal{C}),
\end{aligned}
$$

where the last equation holds because $f=\mathcal{H}_{T}(f)$ at each squared inner product between distinct points in $\mathcal{C}$.

Unfortunately, even if $\mathcal{C}$ is universally optimal, there is no guarantee that it is optimal for the potential functions constructed in Corollary [1] That seems to depend on luck and the proper choice of $T$.

The proof of the main theorem in [CK07] can be recast in this framework, which simplifies the argument given there: specifically, it replaces the use of conductivity. As a side benefit, this approach allows us to give a substantially simpler proof of the universal optimality of the regular 600-cell in $S^{3}$ than was given in [CK07. (That is the one case that was not proved by a conceptual argument, but rather by somewhat complicated calculations.) See Appendix C for more details.

For the rhombic dodecahedron code in $\mathbb{R P}^{2}$, the squared inner products are 0 , $1 / 9$, and $1 / 3$. If we take $T=\{0,0,0,1 / 9,1 / 9,1 / 3,1 / 3\}$ in Corollary 11, we find that it suffices to prove optimality for the potential functions $f(t)=1, t, t^{2}, t^{3}$, $t^{3}(t-1 / 9), t^{3}(t-1 / 9)^{2}$, and $t^{3}(t-1 / 9)^{2}(t-1 / 3)$. Of course the constant function is trivial, and two-point bounds are sharp for $f(t)=t$ (the rhombic dodecahedron code is a projective 1-design). Thus, only five cases remain.

The triple repetition of 0 in $T$ is not essential for the proof, but it is helpful. Our approach fails if we take $\operatorname{mult}_{T}(0)=1$; with $\operatorname{mult}_{T}(0)=2$, it works but the numerical calculations are more cumbersome.

For the rhombic dodecahedron code $\mathcal{C}$,

$$
\widehat{E}_{f}(\mathcal{C})=3 f(0)+6 f(1 / 9)+12 f(1 / 3) .
$$

Thus, we wish to prove a lower bound of $3 f(0)+6 f(1 / 9)+12 f(1 / 3)$ for energy with respect to each of the potential functions $f(t)=t^{2}, t^{3}, t^{3}(t-1 / 9), t^{3}(t-1 / 9)^{2}$, and $t^{3}(t-1 / 9)^{2}(t-1 / 3)$. In fact, in each case we prove something stronger, namely that the same lower bound holds not just for the potential function $f(t)$ but also for

$$
f_{0}(t)=f(t)-\frac{t^{3}(t-1 / 9)^{2}(t-1 / 3)^{2}}{1000} .
$$

Because $f_{0}(t) \leq f(t)$ for all $t \geq 0$, we have $\widehat{E}_{f_{0}} \leq \widehat{E}_{f}$, and equality holds for the rhombic dodecahedron code.

For each potential function, we use an auxiliary function

$$
H(u, v, t)=c+\sum_{k=0}^{5}\left\langle F_{k}, \widehat{T}_{k}^{n}(u, v, t)\right\rangle,
$$


where the matrix $F_{0}$ is $5 \times 5$ (i.e., all entries outside of the upper left $5 \times 5$ block are zero), $F_{1}$ and $F_{2}$ are $4 \times 4, F_{3}$ and $F_{4}$ are $3 \times 3$, and $F_{5}$ is $2 \times 2$. We then optimize the bound obtainable from Theorem 8 . To enforce the constraint that

$$
H(u, v, t) \leq \frac{f_{0}\left(u^{2}\right)+f_{0}\left(v^{2}\right)+f_{0}\left(t^{2}\right)}{3},
$$

we write

$$
\frac{f_{0}\left(u^{2}\right)+f_{0}\left(v^{2}\right)+f_{0}\left(t^{2}\right)}{3}-H(u, v, t)=\langle z(u, v, t), M z(u, v, t)\rangle,
$$

where $M \succeq 0$ is a $120 \times 120$ matrix and $z(u, v, t)$ is the vector consisting of all monomials in $u, v, t$ of degree at most 7 . Note that this condition is very strong (for example, it forces the degree of the left side to be at most 14 and it forces the inequality to hold for all $\left.(u, v, t) \in \mathbb{R}^{3}\right)$, but in fact it works.

The program SDPA-GMP can do arbitrary-precision semidefinite programming, and CSDP can do sufficiently high-precision semidefinite programming for our purposes (see $\mathrm{N}+08$ and $\mathrm{B} 99$, respectively). Using either of them, one can solve this semidefinite program numerically and verify that the bound is nearly sharp. However, getting a rigorous proof takes more work. We would like to round an approximate solution to get an exact solution, but the rounding process can violate the constraints at locations where there is equality, so it must be done carefully.

To get a sharp bound, we will impose two types of conditions. First, there are necessary constraints on the matrices $F_{k}$ from complementary slackness (i.e., the conditions under which equality can hold in the proof of Theorem 8). Specifically, for $0 \leq k \leq 5$, the inner product of $F_{k}$ with

$$
\begin{aligned}
& 35 \delta_{k, 0} J+6 \widehat{T}_{k}^{3}(0,0,0)+24 \widehat{T}_{k}^{3}\left(-\frac{1}{3},-\frac{1}{3},-\frac{1}{3}\right) \\
& +36 \widehat{T}_{k}^{3}\left(-\frac{1}{3}, \frac{1}{\sqrt{3}}, \frac{1}{\sqrt{3}}\right)+72 \widehat{T}_{k}^{3}\left(\frac{1}{\sqrt{3}}, \frac{1}{\sqrt{3}}, 0\right)+72 \widehat{T}_{k}^{3}\left(\frac{1}{3}, \frac{1}{\sqrt{3}}, \frac{1}{\sqrt{3}}\right)
\end{aligned}
$$

must vanish. The coefficients and arguments of $\widehat{T}_{k}^{3}$ come from the triple distribution of the rhombic dodecahedron code. Second, we require that $H(u, v, t)$ must equal $\left(f_{0}\left(u^{2}\right)+f_{0}\left(v^{2}\right)+f_{0}\left(t^{2}\right)\right) / 3$ at the triples of inner products that occur, namely the five arguments to $\widehat{T}_{k}^{3}$ in the formula above, and all their first partial derivatives must agree as well. These conditions are linear in the variables $c, F_{0}, \ldots, F_{5}$, and $M$.

To perform the rounding correctly, we write the problem in a basis for the set of all $c, F_{0}, \ldots, F_{5}$, and $M$ that satisfy the constraints listed in the previous paragraph, together with the defining equations such as

$$
\frac{f_{0}\left(u^{2}\right)+f_{0}\left(v^{2}\right)+f_{0}\left(t^{2}\right)}{3}-H(u, v, t)=\langle z(u, v, t), M z(u, v, t)\rangle .
$$

We then solve the semidefinite program numerically for the coefficients in this basis, and we round the coefficients to eight or nine decimal places to get an exact solution. It is not guaranteed that this rounding process will work: the problem is the semidefinite constraints, because unexpected zero eigenvalues may become negative due to the rounding. However, we do not run into that difficulty, because there turn out to be no zero eigenvalues except for the ones forced by the constraints we have built into our basis. Note that the exact auxiliary function is far from unique, and our rounding strategy makes essential use of this freedom. 
Using this method in each of the five remaining cases proves optimality in Theorem 1. Carrying out the calculations requires a computer, most notably because $M$ is a $120 \times 120$ matrix, but all the calculations are completely rigorous. In particular, we use exact rational arithmetic, with no floating-point approximations. See Appendix D for more details.

To complete the proof, we must verify uniqueness. The Hermite interpolation at more than two points (counting with multiplicity) of an absolutely monotonic polynomial cannot be linear unless the original polynomial is itself linear: otherwise, by Rolle's theorem, the second derivative of their difference would have a root between the points, and hence the second derivative of the original function would vanish, which would violate absolute monotonicity. Thus, to prove uniqueness for nonlinear potential functions, we just need to prove it for the five cases $f(t)=t^{2}$, $t^{3}, t^{3}(t-1 / 9), t^{3}(t-1 / 9)^{2}$, and $t^{3}(t-1 / 9)^{2}(t-1 / 3)$. Let $f(t)$ be one of these, and let $c, F_{0}, \ldots, F_{5}$ and $M$ be as above (for the modified function $f_{0}$ ).

Because $f \geq f_{0}$ pointwise,

$$
H(u, v, t) \leq \frac{f\left(u^{2}\right)+f\left(v^{2}\right)+f\left(t^{2}\right)}{3},
$$

with equality only when $u^{2}, v^{2}, t^{2} \in\{0,1 / 9,1 / 3\}$. Thus, there are only finitely many points at which equality is possible, and we can simply check all the cases. For $(u, v, t) \in D$, the triple $(u, v, t)$ can occur in the global minimum for energy only if equality holds (otherwise, something is lost in the inequalities in the proof of Theorem 8). It follows that the only triples in $D$ that can occur in an optimal configuration are $(0,0,0),(-1 / 3,-1 / 3,-1 / 3),(-1 / 3,1 / \sqrt{3}, 1 / \sqrt{3}),(1 / \sqrt{3}, 1 / \sqrt{3}, 0)$, and $(1 / 3,1 / \sqrt{3}, 1 / \sqrt{3})$, up to equivalence defined by permutations of the coordinates and pairs of sign changes. Let $N_{1}, \ldots, N_{5}$ denote the numbers of times each equivalence class occurs between a triple of distinct points, with the numbering from 1 to 5 in the order given here.

The numbers $N_{1}, \ldots, N_{5}$ must satisfy several linear constraints. Their sum must be 210 , and for $0 \leq k \leq 5$, the inner product of $F_{k}$ with

$$
\begin{aligned}
& 35 \delta_{k, 0} J+N_{1} \widehat{T}_{k}^{3}(0,0,0)+N_{2} \widehat{T}_{k}^{3}\left(-\frac{1}{3},-\frac{1}{3},-\frac{1}{3}\right) \\
& +N_{3} \widehat{T}_{k}^{3}\left(-\frac{1}{3}, \frac{1}{\sqrt{3}}, \frac{1}{\sqrt{3}}\right)+N_{4} \widehat{T}_{k}^{3}\left(\frac{1}{\sqrt{3}}, \frac{1}{\sqrt{3}}, 0\right)+N_{5} \widehat{T}_{k}^{3}\left(\frac{1}{3}, \frac{1}{\sqrt{3}}, \frac{1}{\sqrt{3}}\right)
\end{aligned}
$$

must vanish, for the same complementary slackness reason as above. If we solve these simultaneous equations, we find that $N_{1}=6, N_{2}=24, N_{3}=36, N_{4}=72$, and $N_{5}=72$.

Because $N_{1}=6$, there is a unique triple of orthogonal points, up to permutations. Call them $e_{1}, e_{2}, e_{3}$ (as usual, we deal with arbitrary lifts to the unit sphere). The only possible inner products between points in the code are $0, \pm 1 / \sqrt{3}, \pm 1 / 3$, and \pm 1 , so every point other than $e_{1}, e_{2}, e_{3}$ must have inner product $0, \pm 1 / \sqrt{3}$, or $\pm 1 / 3$ with each $e_{i}$ (note that -1 is ruled out by the uniqueness of $e_{1}, e_{2}, e_{3}$ ). Because $e_{1}, e_{2}, e_{3}$ are orthogonal unit vectors, the sum of the squares of the inner products with them must be 1 . That means they must all be $\pm 1 / \sqrt{3}$. In other words, with respect to the orthonormal basis $e_{1}, e_{2}, e_{3}$, the code consists of the basis vectors together with four points in the cube $( \pm 1 / \sqrt{3}, \pm 1 / \sqrt{3}, \pm 1 / \sqrt{3})$. It follows that it is the rhombic dodecahedron code. 
Finally, for uniqueness as a spherical code, we use $f(t)=t^{3}(t-1 / 9)^{2}(t-1 / 3)$. Every code whose minimal distance is as large as the rhombic dodecahedron code's minimal distance has $\widehat{E}_{f} \leq 0$, and hence it minimizes $\widehat{E}_{f}$ (because the minimal energy is 0 ). Thus, uniqueness for this potential function implies uniqueness as a spherical code. This completes the proof of Theorem 1 .

\section{Open QUESTIONS}

5.1. Three-point bounds and generalizations. One pressing question is why three-point bounds are not sharp more often. Of course, it is unreasonable to expect that sharp bounds will ever be common in packing or energy minimization problems. The phenomena seem to be intrinsically complicated (see, for example, $\mathrm{B}+09$ ), and the most one can hope for is to prove optimality in exceptional cases. However, even just for spheres, the two-point bounds are sharp for three infinite families and a dozen sporadic cases (see Table 1 in [CK07]), and they are sharp even more often in projective spaces. Thus, it is surprising that only three sharp cases are known for three-point bounds, excluding the cases where two-point bounds already suffice. Surely there must be more examples, but so far we have not found them.

It is natural to ask what sort of bounds are required to prove optimality for an $N$-point configuration. Of course $N$-point bounds suffice, but only because in the process of optimizing over $N$-point distributions they implicitly optimize over all $N$ point configurations. For what sorts of families of configurations might $o(N)$-point bounds suffice?

It would be very interesting to do explicit calculations with four-point bounds. This project would involve exceptionally time-consuming calculations, but it is possible in principle and perhaps in practice. Gijswijt, Mittelmann, and Schrijver GMS10, have carried out the analogue for binary error-correcting codes, which suggests that the continuous version may also be tractable.

If we had further examples of sharp three-point bounds, they might suggest organizing principles that could lead to a deeper understanding. For example, for two-point bounds in two-point homogeneous spaces, Levenshtein [L92] proved a beautiful criterion for being an optimal code in terms of strength as a design: any $m$-distance set that is a $(2 m-1)$-design (or even an antipodal ( $2 m-2)$-design) is an optimal code. In these cases, the two-point bounds are sharp and can be understood conceptually, with no need for numerical calculations. The same criterion applies more generally to prove universal optimality (Theorems 1.2 and 8.2 in [CK07]). Every known case in which two-point bounds are sharp fits into this framework, with one exception, namely the regular 600-cell, which is analyzed in [A99, CK07.

We cannot even propose a conjectural generalization of Levenshtein's criterion to three-point bounds. However, we hope that some general principle will explain the sharp cases, offer guidance for how to locate more of them, and lead to proofs that involve no explicit numerical computations.

Another question is whether there are good applications of three-point bounds to potential functions that depend on triples of points, rather than pairs. Of course, there is no obstacle to writing down such bounds, but it is not clear that there are any important examples. Pair potentials are far more common in both physics and mathematics, and we do not know which higher-order generalizations may be worthy of investigation. 
5.2. Projective spaces. Theorem 2 settles the question of universal optimality in $\mathbb{R P}^{2}$, but as we will see in Subsection 5.3 , there may be cases in which three-point bounds are sharp but universal optimality does not hold. We are unaware of any cases in projective space besides the rhombic dodecahedron code in which threepoint bounds are sharp and two-point bounds are not, but it is difficult to imagine that it is the only example.

It is especially intriguing that the three-point bounds prove universal optimality for this code, and it would be fascinating to find other such cases. It is unlikely that there are any in $\mathbb{R P}^{3}$. In addition to the universal optima that occur in every dimension (up to $n$ orthogonal lines in $\mathbb{R}^{n}$, or $n+1$ connecting the vertices of a regular simplex to its centroid), there are at least two other universally optimal line configurations in $\mathbb{R}^{4}: 12$ lines through opposite vertices of a regular 24-cell and 60 from a regular 600-cell. In each of these cases, universal optimality follows from two-point bounds: the last one can be proved using the techniques from Section 7 of CK07, and all the others follow from Theorem 8.2 in CK07. We know of no other candidates for universal optimality in $\mathbb{R P}^{3}$.

Line configurations in $\mathbb{R}^{5}$ are more promising. In addition to the generic cases of up to six lines, two-point bounds prove universal optimality for a ten-line configuration from CHS96] (by Theorem 8.2 in [CK07). In the subspace of $\mathbb{R}^{6}$ consisting of all points with coordinate sum zero, this configuration consists of the lines through all the permutations of $(1,1,1,-1,-1,-1)$. If we include also the lines through the permutations of $(5,-1,-1,-1,-1,-1)$, we get a 16 -line configuration also studied in CHS96. The orthoplex bound is sharp for it, so it is an optimal projective code, but nothing more is known about energy minimization or uniqueness.

Conjecture 12. The 16-line configuration in $\mathbb{R}^{5}$ described above is universally optimal and is the unique optimal 16-point code in $\mathbb{R} \mathbb{P}^{4}$.

The parallels between this code and the rhombic dodecahedron code are noteworthy. The seven lines in $\mathbb{R}^{3}$ can be constructed completely analogously, using the permutations of the points $(1,1,-1,-1)$ and $(3,-1,-1,-1)$. Equivalently, both codes can be constructed by starting with the lines through the vertices of a regular simplex centered at the origin, and then filling in all the holes (i.e., the lines at maximal distance from the original lines).

Both codes can be proved optimal using the orthoplex bound. Nevertheless, the three-point bounds for potential energy do not seem to settle Conjecture 12 , although they suffice to prove Theorem 1, Perhaps four-point bounds can prove Conjecture 12, but the calculations required to investigate this could be formidable. The pattern certainly does not continue to $\mathbb{R}^{7}$ : the analogous configuration has $\left(\begin{array}{l}8 \\ 4\end{array}\right) / 2+8=43$ lines, but comparison with Table 4 from [CHS96] shows that it is not even an optimal projective code.

5.3. Spheres. Although in this paper we focus on real projective space, we have also applied three-point bounds to potential energy minimization on spheres. We have not found any sharp cases beyond the two identified by Bachoc and Vallentin BV09b, namely the Petersen code in $\mathbb{R}^{4}$ (the ten edge midpoints of a regular simplex) and the square antiprism in $\mathbb{R}^{3}$. Using three-point bounds, they proved rigorously that the Petersen code is an optimal code, and based on their calculations they conjectured that the bounds are also sharp for the antiprism. 
For ten points in $S^{3}$, we observe a remarkable phenomenon in the three-point bounds for energy. The Petersen code is not universally optimal, because it sometimes has greater potential energy than the code consisting of two regular pentagons on orthogonal planes in $\mathbb{R}^{4}$. However, the three-point bounds appear to be sharp in every case:

Conjecture 13. For every completely monotonic potential function $f$ on $(0,4]$, either the Petersen code or the orthogonal pentagon code minimizes the energy $E_{f}$ among all ten-point codes in $S^{3}$. Furthermore, the three-point bounds are always sharp (for whichever code is optimal).

In other words, the three-point bounds remain sharp throughout the phase transition between the two ground states. If true, this represents an unprecedented phenomenon in coding and energy minimization.

We believe that Conjecture 13 should be provable, although we have not been able to complete a proof. The cone of completely monotonic functions on $(0,4]$ is spanned by the functions $f(r)=(4-r)^{k}$ for $k=0,1,2, \ldots$ (see Theorem $9 \mathrm{~b}$ in [W41, p. 154]). For $k \leq 2$, both codes have the same energy and the twopoint bounds are sharp. For $3 \leq k \leq 6$, the three-point bounds are sharp for the orthogonal pentagons, and for $k \geq 7$ we can prove that they are sharp for the Petersen code (by using the spherical analogue of Corollary 11 to reduce to a finite basis). To prove Conjecture 13 it would suffice to prove it just for the functions $f(r)=(4-r)^{j}+\alpha_{j, k}(4-r)^{k}$ with $3 \leq j \leq 6$ and $k \geq 7$, where $\alpha_{j, k}>0$ is chosen to make the two codes have equal energy. Unfortunately, we have found it much more difficult to deal with these cases.

The same phenomenon as in Conjecture 13 seems to occur also for antiprisms. In that case, there is a one-parameter family of configurations. Each consists of two squares in parallel planes and offset by a $45^{\circ}$ angle, but the distance between the planes can vary.

Conjecture 14. For every completely monotonic potential function $f$ on $(0,4]$, some square antiprism minimizes the energy $E_{f}$ among all eight-point codes in $S^{2}$. Furthermore, the three-point bounds are always sharp (for whichever code is optimal).

Because this conjecture involves a continuous family of optima, it may be more difficult to prove than Conjecture 13. It seems to require a new idea beyond what suffices for Theorem 1 and our partial progress on Conjecture 13. The dependence of the optimal energy on the potential function is also extraordinarily complicated, because of the need to optimize over all square antiprisms. For example, for the Coulomb potential, the minimal energy within the family of antiprisms is a root of an irreducible polynomial of degree 48 .

These conjectures suggest a broad generalization of the mechanism behind the known proofs of universal optimality. Given a space $X$ and a number of points $N$, call a potential function $k$-point sharp if there exists an $N$-point configuration in $X$ whose energy under that potential function matches the $k$-point bounds. Subject to one technical restriction, we conjecture that all completely monotonic functions are $k$-point sharp if any one of them is:

Conjecture 15. Suppose $X$ is a sphere or projective space and $N \in \mathbb{N}$. If there exists a completely monotonic potential function that is $k$-point sharp for $N$ points 
in $X$ and is not a polynomial, then every completely monotonic potential function is $k$-point sharp for $N$ points in $X$. The same conclusion also holds if the $k$-point bounds prove that an $N$-point configuration is an optimal code in $X$.

For low-degree polynomials, there are numerous counterexamples: for example, for each spherical $k$-design, two-point bounds prove that it is optimal for $f(r)=$ $(4-r)^{k}$. However, that is the only loophole we have found. Conjecture 15] is very strong, and perhaps we have been misled by the few known examples with $k>2$. It may hold in these examples merely because the optimal structures are in some sense of comparable complexity. However, Conjecture 15] seems to be the simplest explanation of the available evidence.

The most dramatic test case will be 24 points in $S^{3}$. The vertices of a regular 24-cell are almost certainly the unique optimal code, but they are not universally optimal [CCEK07]. Instead, there is a one-parameter family of competing configurations that improve on the 24 -cell for some potential functions. Numerical evidence suggests that either the 24-cell or one of these competitors is always optimal. If that is true, and Conjecture [15] is true as well, then $k$-point bounds cannot settle the optimality of the 24-cell without also dealing with its more exotic competitors. If they can accomplish both with a small value of $k$, it will be truly remarkable.

\section{Appendix A. The orthoplex Bound}

In this appendix, we explain the orthoplex bound from [CHS96] in somewhat different terms than those used there (although it is essentially the same approach), and we discuss some examples and generalizations.

The orthoplex bound applies to codes in any Grassmannian, but for real projective space it is particularly simple. It is based on the map from $\mathbb{R}^{n}$ to its symmetric square $\operatorname{Sym}^{2} \mathbb{R}^{n}$ that takes $x$ to $x \otimes x$. Under the natural inner product on $\mathrm{Sym}^{2} \mathbb{R}^{n},\langle x \otimes x, y \otimes y\rangle=\langle x, y\rangle^{2}$. Let $e_{1}, \ldots, e_{n}$ be an orthonormal basis of $\mathbb{R}^{n}$, and let $E=\sum_{i=1}^{n} e_{i} \otimes e_{i}$. Then for all $x \in \mathbb{R}^{n},|x|^{2}=\langle x \otimes x, E\rangle$. Thus, the unit sphere $S^{n-1}$ is mapped into the cross section of the unit sphere in $\mathrm{Sym}^{2} \mathbb{R}^{n}$ that has inner product 1 with the vector $E$ (note that $|E|^{2}=n$ ).

For $x \in S^{n-1}$, define $\varphi(x)=(x \otimes x-E / n) / \sqrt{1-1 / n}$. (In other words, start with $x \otimes x$, project orthogonally to $E$, and rescale to get a unit vector.) Then $|\varphi(x)|^{2}=1$ and $\langle\varphi(x), \varphi(y)\rangle=\left(\langle x, y\rangle^{2}-1 / n\right) /(1-1 / n)$, which implies that $\varphi(x)=\varphi(y)$ if and only if $x= \pm y$. The image of $\varphi$ is orthogonal to $E$ and is thus contained in the unit sphere of a Euclidean space of $n(n+1) / 2-1$ dimensions.

Under $\varphi$, an $(n, N, t)$ antipodal code is mapped to an

$$
\left(n(n+1) / 2-1, N / 2,\left(t^{2}-1 / n\right) /(1-1 / n)\right)
$$

spherical code. Thus, any bound on such spherical codes yields a bound on the original antipodal code. Linear programming bounds are useless in this context, because any positive-definite function on the higher-dimensional sphere pulls back under $\varphi$ to a positive-definite function on $S^{n-1}$ (so there is no need to apply $\varphi$ in the first place). However, other bounds may prove fruitful. For example, for $m+1<k \leq 2 m$, no $k$-point code in $S^{m-1}$ can achieve an angle of better than $\pi / 2$ (see [R55] or Theorem 6.2.1 in [B04]). It follows that when $n(n+1) / 2<$ $N / 2 \leq n(n+1)-2$, no $N$-point antipodal code in $S^{n-1}$ can have maximal inner product less than $1 / \sqrt{n}$, since otherwise its image under $\varphi$ would have maximal inner product less than 0 . This is the orthoplex bound for projective codes. 
It is not obvious that the orthoplex bound is ever sharp, but in fact it is sharp in several important cases, for example when

$$
(N / 2, n) \in\{(7,3),(11,4),(12,4),(16,5),(22,6),(46,9),(47,9),(48,9)\} .
$$

These cases are all taken from CHS96. The $(7,3)$ case is of course the rhombic dodecahedron code. Another noteworthy case is $N / 2=n(n+2) / 2$ with $n$ a power of 4 (which generalizes the $(12,4)$ case), due to Levenshtein [L82. In this case, the orthoplex bound shows that optimality still holds when up to $n / 2-1$ lines are omitted.

The orthoplex bound does not imply uniqueness. One problem is that when $N / 2<n(n+1)-2$, there are continuous families of spherical codes with maximal inner product 0 . To prove uniqueness, one would have to determine which such codes are in the image of $\varphi$, which seems to be a subtle question. Even when $N / 2=n(n+1)-2$, the image of $\varphi$ is uniquely determined up to isometries of the sphere containing it, but it does not obviously follow that the original code is also unique.

Uniqueness is not merely difficult to prove, but in fact sometimes false. For example, the unique $(4,24,1 / 2)$ antipodal code is the regular 24-cell (uniqueness follows easily from the two-point bounds). As one can see in the list of examples above, the orthoplex bound proves not only that it is optimal, but also that removing one pair of antipodal points still yields an optimal antipodal code of size 22 . However, that is not the only $(4,22,1 / 2)$ antipodal code. Another one is given by the vertices $( \pm 1 / \sqrt{3}, \pm 1 / \sqrt{3}, \pm 1 / \sqrt{3}, 0)$ of a cube, a pair $(0,0,0, \pm 1)$ of points antipodal to all of them, and the two octahedra

$$
( \pm \sqrt{3} / 2,0,0, \pm 1 / 2), \quad(0, \pm \sqrt{3} / 2,0, \pm 1 / 2), \quad(0,0, \pm \sqrt{3} / 2, \pm 1 / 2)
$$

in the 3 -dimensional affine subspaces with fourth coordinate $1 / 2$ or $-1 / 2$. This example, which appears to be new, is a $(4,22,1 / 2)$ antipodal code that is not contained in the 24-cell, because inner products of $\pm 1 / 3$ and $\pm 1 / 4$ occur. We do not know of any other $(4,22,1 / 2)$ antipodal codes, but we have not searched thoroughly enough to be confident that no others exist.

Note that the second Gegenbauer polynomial $P_{2}^{n}$ for $S^{n-1}$ is given by $P_{2}^{n}(t)=$ $\left(t^{2}-1 / n\right) /(1-1 / n)$. Thus, the map $\varphi$ satisfies $\langle\varphi(x), \varphi(y)\rangle=P_{2}^{n}(\langle x, y\rangle)$. This is not a coincidence, and in fact the orthoplex bound can be generalized to substitute any positive-definite function $f$ for $P_{2}^{n}$ if it is normalized to satisfy $f(1)=1$. For individual Gegenbauer polynomials this follows immediately from the reproducing kernel property (see Subsection 2.2 of [CK07] for a review of the theory), and nonnegative linear combinations can be obtained by taking direct sums of the corresponding spaces. Unfortunately, we have been unable to find any cases other than $f=P_{2}^{n}$ that lead to sharp bounds on antipodal or general spherical codes.

\section{Appendix B. Universal optima in $\mathbb{R P}^{2}$}

The proof of completeness for the list in Theorem 2 is based on a theorem of Leech [L57]. He classified balanced configurations in $S^{2}$, i.e., point configurations that are in equilibrium for every pairwise force law depending only on distance. Equivalently, in the space of configurations they are critical points for energy for 
TABLE 1. Balanced configurations that must be eliminated.

\begin{tabular}{lcccc} 
Polyhedron & Combination & $N$ & Cosine of minimal angle & Record \\
\hline Octahedron & $\mathrm{E}$ & 6 & $1 / 2$ & $0.447 \ldots$ \\
Octahedron & $\mathrm{VE}$ & 9 & $1 / \sqrt{2}=0.707 \ldots$ & $0.669 \ldots$ \\
Octahedron & $\mathrm{EF}$ & 10 & $\sqrt{2 / 3}=0.816 \ldots$ & $0.686 \ldots$ \\
Octahedron & $\mathrm{VEF}$ & 13 & $\sqrt{2 / 3}=0.816 \ldots$ & $0.768 \ldots$ \\
Icosahedron & $\mathrm{E}$ & 15 & $(1+\sqrt{5}) / 4=0.809 \ldots$ & $0.786 \ldots$ \\
Icosahedron & $\mathrm{F}$ & 10 & $\sqrt{5} / 3=0.745 \ldots$ & $0.686 \ldots$ \\
Icosahedron & $\mathrm{VE}$ & 21 & $\sqrt{(5+\sqrt{5}) / 10}=0.850 \ldots$ & $0.846 \ldots$ \\
Icosahedron & $\mathrm{VF}$ & 16 & $\sqrt{(5+2 \sqrt{5}) / 15}=0.794 \ldots$ & same \\
Icosahedron & $\mathrm{EF}$ & 25 & $\sqrt{(3+\sqrt{5}) / 6}=0.934 \ldots$ & $0.872 \ldots$ \\
Icosahedron & $\mathrm{VEF}$ & 31 & $\sqrt{(3+\sqrt{5}) / 6}=0.934 \ldots$ & $0.894 \ldots$
\end{tabular}

every potential function. This is a necessary condition for universal optimality in $S^{2}$, and for universal optimality in $\mathbb{R P}^{2}$ it is necessary that the corresponding antipodal configuration in $S^{2}$ be balanced. (If it is not balanced, then the net forces inherit the antipodal symmetry and descend to projective space.)

One obvious balanced configuration is a ring of equally spaced points around the equator, and it remains balanced if one includes the north and south poles as well. In addition, each regular polyhedron gives several balanced configurations. Its vertices, face centers, and edge midpoints each form a balanced configuration, and every combination of them (vertices and faces, vertices and edges, faces and edges, or all three) is also balanced when they are rescaled to lie on the same sphere. Leech proved that this list of balanced configurations in $S^{2}$ is complete. By contrast, the high-dimensional case is not so simple, and there exist balanced configurations with no symmetry at all [CEKS10].

To classify the universal optima in $\mathbb{R P}^{2}$, one need only examine the antipodal balanced configurations in $S^{2}$. The cases with a ring around the equator are easily dealt with: whenever the ring has more than four points in it and the potential function is sufficiently steep, one can lower the energy by moving one point slightly off the equator (and of course moving the antipodal point correspondingly). The only difficulty is dealing with the finitely many cases that come from regular polyhedra.

Table 1 shows a list of the cases that must be eliminated. It omits the actual universal optima from Theorem 2 as well as any duplicate cases (for example, from duality). The first four columns specify the polyhedron, which combination of vertices, edges, and faces is being used, the number $N$ of lines, and the cosine of the minimal angle between them. The final column tells the smallest such cosine known for a configuration of this size, from Table 1 in CHS96. In every case except $N=16$, the record from CHS96 is better than the balanced configuration, which is therefore not universally optimal since it is not even an optimal projective code. However, the balanced configuration with $N=16$ is probably an optimal code.

Nevertheless, the $N=16$ case is not universally optimal. It is a 2-design in $\mathbb{R P}^{2}$ but not a 3 -design. However, a 16-point 3-design exists: it is equivalent to 
an antipodal 32-point spherical 7-design in $S^{2}$, and such a design is constructed in Section 4 of [HS96]. It follows that the 16-line configuration is not universally optimal, and hence the list in Theorem 2 is complete.

\section{Appendix C. Universal optimality of the Regular 600-CELL}

Theorem 16 (Cohn and Kumar CK07]). The 120 vertices of the regular 600-cell are universally optimal in $S^{3}$.

Sketch of proof. Section 7 of [CK07] describes a construction that applies two-point bounds for energy. It gives a sharp bound for every absolutely monotonic potential function, and in any given case it is easy to check that it works, but the proof that it works for all of them is fairly elaborate. We will use Corollary 11(or, technically, its analogue for spheres) to reduce the problem to a finite calculation.

There are eight inner products between distinct points in the 600-cell, namely $-1,0, \pm 1 / 2$, and $( \pm 1 \pm \sqrt{5}) / 4$. Call them $t_{1}, \ldots, t_{8}$ with $-1=t_{1}<t_{2}<\cdots<t_{8}$. Let $T$ be the multiset $\left\{t_{1}, t_{2}, t_{2}, t_{3}, t_{3}, \ldots, t_{8}, t_{8}\right\}$ (note that $\operatorname{mult}_{T}\left(t_{1}\right)=1$ ). Now Corollary 11 reduces the problem to a finite number of cases. In each case but the last, it is not hard to check that the approach in Section 7 of [CK07] proves a sharp bound, but it fails in the last case, namely

$$
f(t)=\left(t-t_{1}\right)\left(t-t_{2}\right)^{2} \ldots\left(t-t_{7}\right)^{2}\left(t-t_{8}\right) .
$$

The failure does not come as a surprise, because of course this function is not absolutely monotonic. Nevertheless, the approach can be salvaged in this case. In the language used at the top of page 127 in [CK07, one should choose an auxiliary polynomial of degree 18 that agrees with $f$ to order 2 at -1 (in addition to the other conditions listed in CK07]). It is then straightforward to check that this polynomial proves a sharp bound for $\widetilde{E}_{f}$.

This proof is still not quite conceptual, because it relies on a fair amount of computation, but it requires less computation and is substantially simpler than the proof in CK07. The same approach of using the spherical analogue of Corollary 11 works for all the universal optima studied in CK07; in fact, it is in a sense equivalent to the approach used there, but this method was not made explicit in CK07. and was not applied to the 600-cell.

\section{Appendix D. Computer CAlCulations}

Computer algebra files for checking the proof of Theorem 1 can be downloaded as part of the source files for this paper in the arXiv.org e-print archive, where it is available as arXiv:1103.0485. In addition to the $\mathrm{AT}_{\mathrm{E}} \mathrm{X}$ source for the paper, downloading the source files produces eight plain text files: definitions.txt, optimal.txt, unique.txt, and data1.txt through data5.txt. These files contain code for the PARI/GP computer algebra system [PARI], which is freely available. The code is not especially elaborate and should be straightforward to adapt to other systems. The files are also available from http://dx.doi.org/10.1090/ S0894-0347-2012-00737-1.

Each of the data files contains the exact values of $c, F_{0}, \ldots, F_{5}$, and $M$ for one of the five potential functions considered in the proof of Theorem 1 . Specifically, it 
defines a constant $c$ and a list $L$ of six matrices, namely $F_{0}, \ldots, F_{5}$, and $M$. Note that in PARI format, $[a, b ; c, d]$ denotes the matrix

$$
\left(\begin{array}{ll}
a & b \\
c & d
\end{array}\right)
$$

In the proof of the theorem, we did not specify the order of the monomials $u^{i} v^{j} t^{k}$ in the vector $z(u, v, t)$, or equivalently the order of the rows and columns of $M$; in the data files, we order them lexicographically by their exponent vectors $(i, j, k)$.

The file definitions.txt defines the ultraspherical polynomials and the matrices $\widehat{S}_{k}^{n}$ and $\widehat{T}_{k}^{n}$. These definitions are read by the remaining two files.

The file optimal.txt completes the proof of universal optimality. Specifically, it checks that all the matrices are symmetric and positive semidefinite, it checks the sum of squares decomposition

$$
\frac{f_{0}\left(u^{2}\right)+f_{0}\left(v^{2}\right)+f_{0}\left(t^{2}\right)}{3}-H(u, v, t)=\langle z(u, v, t), M z(u, v, t)\rangle,
$$

and it checks that the bound is sharp. To show that a $d \times d$ matrix is positive semidefinite, it computes the characteristic polynomial $p(x)$ and verifies that $(-1)^{d} p(-x)$ has no negative coefficients. All the calculations are done using exact rational arithmetic, so the resulting proof is rigorous.

Finally, the file unique.txt completes the proof of uniqueness and thus of Theorem 1. It finds all $(u, v, t)$ such that

$$
\frac{f\left(u^{2}\right)+f\left(v^{2}\right)+f\left(t^{2}\right)}{3}=H(u, v, t),
$$

and it solves a system of simultaneous equations to determine how many times such a triple must occur in an optimal configuration (i.e., to compute the numbers $\left.N_{1}, \ldots, N_{5}\right)$. It is convenient to use the number $1 / \sqrt{3}$, but we must avoid floating point arithmetic to keep round-off error from becoming a problem. To do so, we use polynomials in a variable $s$ and work modulo $s^{2}-1 / 3$. As above, all the calculations are then carried out exactly.

\section{ACKNOWLEDGEMENTS}

We thank Christine Bachoc, Abhinav Kumar, and Frank Vallentin for helpful discussions and Nathan Kaplan and the anonymous referees for their feedback on the manuscript.

\section{REFERENCES}

[A99] N. N. Andreev, A spherical code, Russian Math. Surveys 54 (1999), no. 1, 251-253. MR:1706807

[AAR99] G. Andrews, R. Askey, and R. Roy, Special Functions, Encyclopedia Math. Appl. 71, Cambridge University Press, Cambridge, 1999. MR.1688958 (2000g:33001)

[B06] C. Bachoc, Linear programming bounds for codes in Grassmannian spaces, IEEE Trans. Inform. Theory 52 (2006), 2111-2125, arXiv:math.C0/0610812. MR2234468 (2007h:94095)

[BV08] C. Bachoc and F. Vallentin, New upper bounds for kissing numbers from semidefinite programming, J. Amer. Math. Soc. 21 (2008), 909-924, arXiv:math/0608426. MR.2393433 (2009c:52029)

[BV09a] C. Bachoc and F. Vallentin, Semidefinite programming, multivariate orthogonal polynomials, and codes in spherical caps, Europ. J. Comb. 30 (2009), 625-637, arXiv:math/0610856. MR2494437 (2010d:90065) 
[BV09b] C. Bachoc and F. Vallentin, Optimality and uniqueness of the $(4,10,1 / 6)$ spherical code, J. Comb. Theory Ser. A 116 (2009), 195-204, arXiv:0708.3947. MR2469257 (2010f:94337)

[B+09] B. Ballinger, G. Blekherman, H. Cohn, N. Giansiracusa, E. Kelly, and A. Schürmann, Experimental study of energy-minimizing point configurations on spheres, Experiment. Math. 18 (2009), 257-283, arXiv:math.MG/0611451. MR2555698 (2010j:52037)

[B41] S. Bochner, Hilbert distances and positive definite functions, Ann. of Math. (2) 42 (1941), 647-656. MR0005782(3:206d)

[B99] B. Borchers, CSDP, a C library for semidefinite programming, Optim. Methods Softw. 11 (1999), 613-623. MR.1778432

[B04] K. Böröczky, Jr., Finite packing and covering, Cambridge Tracts in Mathematics 154, Cambridge University Press, Cambridge, 2004. MR2078625 (2005g:52045)

[BG09] M. Bowick and L. Giomi, Two-dimensional matter: order, curvature and defects, Advances in Physics 58 (2009), 449-563, arXiv:0812.3064.

[C10] H. Cohn, Order and disorder in energy minimization, Proceedings of the International Congress of Mathematicians, Hyderabad, August 19-27, 2010, Volume IV, pages 24162443, Hindustan Book Agency, New Delhi, 2010, arXiv:1003.3053. MR2827978

[CCEK07] H. Cohn, J. H. Conway, N. D. Elkies, and A. Kumar, The D4 root system is not universally optimal, Experiment. Math. 16 (2007), 313-320, arXiv:math.MG/0607447. MR.2367321 (2008m:52041)

[CEKS10] H. Cohn, N. D. Elkies, A. Kumar, and A. Schürmann, Point configurations that are asymmetric yet balanced, Proc. Amer. Math. Soc. 138 (2010), 2863-2872, arXiv:0812.2579. MR2644899(2011d:52021)

[CK07] H. Cohn and A. Kumar, Universally optimal distribution of points on spheres, J. Amer. Math. Soc. 20 (2007), 99-148, arXiv:math.MG/0607446. MR2257398 (2007h:52009)

[CHS96] J. H. Conway, R. H. Hardin, and N. J. A. Sloane, Packing lines, planes, etc.: packings in Grassmannian spaces, Experiment. Math. 5 (1996), 139-159. MR.1418961 (98a:52029)

[CS99] J. H. Conway and N. J. A. Sloane, Sphere Packings, Lattices and Groups, third edition, Grundlehren Math. Wiss. 290, Springer-Verlag, New York, 1999. MR.1662447 (2000b:11077)

[D72] P. Delsarte, Bounds for unrestricted codes, by linear programming, Philips Res. Reports 27 (1972), 272-289. MR0314545 (47:3096)

[GMS10] D. C. Gijswijt, H. D. Mittelmann, and A. Schrijver, Semidefinite code bounds based on quadruple distances, to appear in IEEE Trans. Inform. Theory, arXiv: 1005.4959.

[HS96] R. H. Hardin and N. J. A. Sloane, McLaren's improved snub cube and other new spherical designs in three dimensions, Discrete Comput. Geom. 15 (1996), 429-441. MR 1384885 (97b:52013)

[L85] S. Lang, $\mathrm{SL}_{2}(\mathbb{R})$, Graduate Texts in Mathematics 105, Springer-Verlag, New York, 1985. MR 803508 (86j:22018)

[L57] J. Leech, Equilibrium of sets of particles on a sphere, Math. Gaz. 41 (1957), 81-90. MR0086325 (19:165b)

[L82] V. I. Levenshtein, Bounds for the maximal cardinality of a code with bounded modulus of the inner product (Russian), Dokl. Akad. Nauk SSSR 263 (1982), 1303-1308; translation in Soviet Math. Doklady 25 (1982), 526-531. MR653223 (83j:94025)

[L92] V. I. Levenshtein, Designs as maximum codes in polynomial metric spaces, Acta Appl. Math. 29 (1992), 1-82. MR1192833 (93j:05012)

[M08] M. Marshall, Positive polynomials and sums of squares, Math. Surveys Monogr. 146, American Mathematical Society, Providence, RI, 2008. MR2383959 (2009a:13044)

[M07] O. Musin, Multivariate positive definite functions on spheres, preprint, 2007, arXiv:math/0701083.

[N+08] M. Nakata, B. J. Braams, K. Fujisawa, M. Fukuda, J. K. Percus, M. Yamashita, and Z. Zhao, Variational calculation of second-order reduced density matrices by strong $N$ representability conditions and an accurate semidefinite programming solver, J. Chem. Phys. 128 (2008), 164113:1-14.

[PARI] PARI/GP, version 2.3.4, Bordeaux, 2008, http://pari.math.u-bordeaux.fr/.

[P44] A. L. Patterson, Ambiguities in the X-ray analysis of crystal structures, Phys. Rev. 65 (1944), 195-201. 
[P07] F. Pfender, Improved Delsarte bounds for spherical codes in small dimensions, J. Combin. Theory Ser. A 114 (2007), 1133-1147, arXiv:math/0501493. MR:2337242 (2009g:94144)

[P93] M. Putinar, Positive polynomials on compact semi-algebraic sets, Indiana Univ. Math. J. 42 (1993), 969-984. MR.1254128 (95h:47014)

[R55] R. A. Rankin, The closest packing of spherical caps in $n$ dimensions, Proc. Glasgow Math. Assoc. 2 (1955), 139-144. MR0074013 (17:523c)

[S42] I. J. Schoenberg, Positive definite functions on spheres, Duke Math. J. 9 (1942), 96108. MR0005922 (3:232c)

[S05] A. Schrijver, New code upper bounds from the Terwilliger algebra and semidefinite programming, IEEE Trans. Inform. Theory 51 (2005), 2859-2866. MR2236252 (2007a:94147)

[SW51] K. Schütte and B. L. van der Waerden, Auf welcher Kugel haben 5, 6, 7, 8 oder 9 Punkte mit Mindestabstand Eins Platz?, Math. Ann. 123 (1951), 96-124. MR0042150 $(13: 61 \mathrm{e})$

[W41] D. V. Widder, The Laplace Transform, Princeton Math. Ser. 6, Princeton University Press, Princeton, NJ, 1941. MR.0005923 (3:232d)

[Y92] V. A. Yudin, Minimum potential energy of a point system of charges (Russian), Diskret. Mat. 4 (1992), 115-121; translation in Discrete Math. Appl. 3 (1993), 7581. MR1181534 (93f:31008)

Microsoft Research New England, One Memorial Drive, Cambridge, Massachuetts 02142

E-mail address: cohn@microsoft.com

Department of Mathematics, Harvard University, Cambridge, Massachusetts 02138

E-mail address: woo@math.harvard.edu 